\title{
Islamische Gemeinden in Deutschland: Strukturen und Angebote ${ }^{1}$
}

\section{Fragestellung}

Das gewachsene Interesse am Islam und an den Muslimen sowie an der Einwandererintegration hat in der vergangenen Dekade zu einer intensiven sozialwissenschaftlichen Auseinandersetzung mit diesen Themen geführt. Dabei steht häufig speziell der organisierte Islam im Mittelpunkt, der die Interessen der Gläubigen artikuliert und seitens der Politik und der Medien zumeist als Ansprechpartner für Fragen des Zusammenlebens begriffen wird, ohne dass jedoch bisher ein tieferer Einblick in die muslimische Organisationslandschaft in Deutschland insgesamt erarbeitet worden wäre. Dies leistet der vorliegende Aufsatz, der auf der Befragung von 1.141 muslimischen (einschließlich alevitischen) Gemeinden beruht. Dabei galt es nicht nur, grundlegende Informationen über die Gemeinden (Größe, Glaubensrichtung usw.) zu erheben, sondern auch, die Anschlussfähigkeit der Studie an die gesellschaftspolitische Debatte um die »Integration « des Islams und der Muslime zu gewährleisten.

Die Integrationsbilanz ist, so die öffentliche Wahrnehmung, defizitär. Tatsächlich zeigen verschiedene empirische Studien eine klare Unterprivilegierung des muslimischen Bevölkerungsteils in Deutschland, insbesondere mit Blick auf die Bildungsbeteiligung, den Berufsstatus und Einkommensindikatoren. Zugleich ist diese Unterprivilegierung als gravierender einzuschätzen als andere Defizite der Sozialintegration (Kontakte zur Aufnahmegesellschaft, räumliche Segregation etc.), ${ }^{2}$ die aber in viel größerem Umfang als die Unterprivilegierung Gegenstand aufgeregter Desintegrationsdebatten geworden sind. Jenseits der individuellen Sozialintegration der Muslime ist der Islam in Deutschland auch nur unzulänglich institutionalisiert und genießt nicht die Rechte, die anderen Religionsgemeinschaften zukommen. ${ }^{3}$ Entsprechend formuliert die Deutsche Islamkonferenz (DIK) ${ }^{4}$ als Ziel »eine bessere institutionelle und gesellschaftliche Integration der rund vier Millionen Muslime in

1 Die vorliegenden Ergebnisse entstammen einem 2010-2012 durch den Europäischen Integrationsfond (EIF) und das Bundesamt für Migration und Flüchtlinge (BAMF) geförderten Projekt. Wir danken Siamak Asgari und Thorsten Amberge für ihre Hilfe bei der Durchführung sowie Cem Sentürk für die Mitarbeit bei der Erstellung der Datenbank muslimischer Organisationen in Deutschland.

2 Übereinstimmend Haug et al. 2009, S. 344, und Sauer, Halm 2009, S. 119-122.

3 Siehe hierzu Azzaoui 2011.

4 In der 17. Legislaturperiode in ihrer 2. Auflage.

Leviathan, 40. Jg., 1/2012, S.71 7108 
Deutschland $\aleph^{5}$, wobei im sozialintegrativen Bereich eine besondere Betonung auf dem Genderthema und der Bekämpfung von Islamismus und Fremdenfeindlichkeit liegt.

Der vorliegende Text greift speziell drei Aspekte der öffentlichen und wissenschaftlichen Debatte um die Sozialintegration der Muslime und die systemische Integration des Islams heraus und leistet einen Beitrag zur Verbesserung der empirischen Grundlage, auf der diese Aspekte diskutiert werden:

- das Verhältnis zwischen eher assimilations- und eher binnenintegrationsorientierten Angeboten der Gemeinden,

- die Bedeutung der Verbände für den organisierten Islam sowie

- die Folgen intergenerationalen Wandels für die Organisationslandschaft, einschließlich des Aspekts (sich eventuell wandelnder) Herkunfts- bzw. Ankunftslandorientierung der Gemeinden.

Im Hauptteil des Aufsatzes wird auch der Frage nachgegangen, ob diese sich auf die Gemeinden beziehenden Debatten für die Integrationspolitik praktisch überhaupt von Belang sind.

Der vorliegende Beitrag soll Anhaltspunkte für die Beurteilung des Beitrags der Gemeinden zur Sozialintegration liefern und zugleich darstellen, welche möglichen Wege die weitere Etablierung des Islams und seiner Organisationen in Deutschland nehmen kann, um den Muslimen ebenso wie den aufnahmegesellschaftlichen Akteuren nachhaltige Förderstrategien aufzuzeigen.

\section{Debatten um die Integration des Islams und der Muslime in Deutschland}

\subsection{Integrationsverständnis und Angebote der Gemeinden}

Bei der Beschäftigung mit dem Thema »Integration « von Einwanderern stellt sich das Problem des zugrunde liegenden Integrationsbegriffs. Wenn sich beispielsweise öffentliche Konflikte um Moscheebauten entzünden, stellt sich die Frage, ob diese Form der eigenkulturellen Vergesellschaftung nicht mit sozialen Desintegrationsentwicklungen im Zusammenhang steht, die häufig unter das Schlagwort der »Parallelgesellschaft " gefasst werden. ${ }^{6}$ Der Anschluss an diese diffuse, dem Alltagsdiskurs folgende Debatte in einem wissenschaftlichen Text ist nicht einfach herzustellen. Und selbst der Bezug auf die Kriterien der Migrationsforschung für gelingende Sozialintegration löst das Problem nicht wirklich, erweisen sich doch die von Hartmut Esser etablierten Dimensionen der Sozialintegration ${ }^{7}$ (Akkulturation, gesellschaftliche Platzierung, Interaktion und Identifikation mit der Aufnahmegesellschaft) bzw. die Zusammenhänge zwischen diesen Dimensionen in der Empirie als deutlich kontextabhängig. ${ }^{8}$ Zugleich bestehen grundsätzliche Einwände dahinge-

5 Bundesministerium des Innern 2011.

6 Leggewie 2009, S. 117-118.

7 Siehe zu diesen Dimensionen und ihrer empirischen Bedeutung Esser 2009.

8 Siehe zu dieser Diskussion Crul, Schneider 2010. 
hend, ob Vorstellungen der Integration von Einwanderern in national definierte Gesellschaften, in denen so etwas wie ein Hauptmuster kenntlich wird, auf das sich die Sozialintegration beziehen kann, die Lebenswirklichkeit von Einwanderern noch abbilden können ${ }^{9}$ und ob nicht multiple Integrationsformen (etwa in die Herkunftsund Aufnahmelandkultur) zur Normalität werden. ${ }^{10}$ Berücksichtigt man alle diese Optionen, so führt dies zwangsläufig zu einem weit gefassten Verständnis möglicher sozialintegrativer Leistungen der hier befragten Gemeinden. Dieser extensive Integrationsbegriff wird aber erst dann zum Problem, wenn eher auf Assimilation abzielende (etwa deutsche Sprachkurse für Jugendliche) und binnenintegrative Angebote (etwa Freizeitangebote, die ausschließlich die eigenethnische Bevölkerung ansprechen) sich in den Gemeinden als konkurrierend erweisen. Geht beides Hand in Hand, bedarf das Verhältnis von Binnenintegration und Assimilation bei der Beurteilung der Angebote im hiesigen Kontext keiner weiteren Problematisierung. Neuere Befunde zur Sozialintegration von Einwanderern in Deutschland stützen diese Überlegung und weisen eher auf ein neutrales denn auf ein Konkurrenzverhältnis von Assimilation und Binnenintegration hin. ${ }^{11}$

Quer zur öffentlichen Debatte um die (des)integrative Wirkung von Migrantenorganisationen liegt die Betrachtung von Netzwerkbildung und Sozialkapital: ${ }^{12}$ So sind bei Portes ${ }^{13}$ die unterschiedlichen Sozialkapitalformen untrennbar miteinander verbunden. Eine Gesellschaft, die ausschließlich assimilative Angebote zur Integration in eine (kulturell homogene) Majorität produzieren würde, wäre desintegrativ mit Blick auf die Einwanderer und würde deren Einbezug verunmöglichen. Integrationsbeiträge der Gemeinden wären vor dem Hintergrund dieser Überlegung vor allem an der Vielfalt der Angebote und deren Offenheit zu messen.

Verbreitet ist die Annahme, die Glaubensrichtung habe Einfluss auf die Offenheit und das Integrationsengagement der Gemeinden, wobei etwa alevitische Organisationen positiver eingeschätzt werden als sunnitische und andere. ${ }^{14}$ Dem bosnischen Islam wird sogar eine spezifisch europäische Prägung - also die Entwicklung eines eigenen Diaspora-Islam - zugeschrieben. ${ }^{15}$ Ebenso wird oft davon ausgegangen, dass Moscheen unterschiedlicher Verbände unterschiedliche Grade von Offenheit oder Integrationsorientierung aufweisen, ${ }^{16}$ ohne dass solche Annahmen bisher empirisch und systematisch vergleichend erhärtet werden konnten. Der organisationssoziologische Blick auf die Gemeinden würde zeigen: Die meisten Angebote erfor-

9 Dieses Argument bei Alba, Nee 2003.

10 Siehe Esser 2008.

11 Esser 2009.

12 Siehe zur Anwendung dieser Konzepte auf Migrantenorganisationen Thränhardt, Hunger 2000 und Thränhardt, Weiss 2005.

13 Portes 1998.

14 Vgl. Sökefeld 2008 c, S. 278.

15 Siehe etwa Homeyer 2007.

16 Man denke an die Beobachtung der Islamischen Gemeinschaft Milli Görüşs (IGMG) durch den Verfassungsschutz. 
dern materielle und immaterielle Ressourcen und setzen zudem ein gewisses Maß an Möglichkeiten zur Aufgabendifferenzierung voraus. Die Öffnung gegenüber unterschiedlichen Gruppen kann ebenso ressourcenabhängig sein. Möglicherweise besteht vor diesem Hintergrund dann doch eine Konkurrenz zwischen religiösen und nichtreligiösen, sozialintegrativen Angeboten. ${ }^{17}$

\subsection{Bedeutung der Verbände}

Bei den Verbänden, in denen ein Teil der von uns befragten Gemeinden organisiert ist, stellt sich die Frage, für wie viele Gemeinden und Gläubige sie jeweils sprechen und wie viele Moscheegemeinden (mit wie vielen Mitgliedern) nicht in den (etablierten) Verbänden vertreten sind. Diese Frage ist zweifellos wichtig, um überhaupt abzuschätzen, von welchen bestehenden Strukturen eine wie auch immer geartete weitere Institutionalisierung des Islams in Deutschland auszugehen hätte. Manche Beobachter sehen in der auch im Rahmen der DIK immer wieder auftretenden Frage nach der Vertretungsmacht der Verbände aber ebenso den Versuch der Relativierung der von ihnen repräsentierten, eher konservativen Positionen zugunsten einer autochthonen europäischen Variante, der es jedoch wiederum weitgehend an Organisationsstrukturen fehlt. ${ }^{18}$ Die Repräsentation der Muslime durch die Verbände wird damit zu einer politischen Frage, die eng mit der wissenschaftlichen Forschung zum Islam verbunden ist.

In den letzten Jahren wurden mehrere groß angelegte, repräsentative Bevölkerungsbefragungen von Muslimen in Deutschland durchgeführt, ${ }^{19}$ die mitunter auch detailliert die Zugehörigkeit der Befragten zu Glaubensrichtungen oder muslimischen Organisationen thematisieren. ${ }^{20}$ Weiterhin sind Versuche der Bestandsaufnahme von Migrantenorganisationen im Allgemeinen ${ }^{21}$ oder von muslimischen Or-

17 Vgl. zu diesem Argument Pugh et al. 1969.

18 So die Position von Azzaoui 2011, S. 262, der sich noch auf weitere, auch aufnahmegesellschaftliche Autoren bezieht.

19 Siehe Brettfeld, Wetzels 2007; Bertelsmann-Stiftung 2008.

20 Siehe Haug et al. 2009. In Deutschland ist die Mehrheit von 74 \% der Muslime sunnitisch, gefolgt von den Aleviten mit $13 \%$ und Schiiten mit einem Anteil von $7 \%$. Daneben gibt es eine recht große Gruppe der Ahmadiyya (ca. 2 \%; ebd., S. 79). Unter den Sunniten in Deutschland ist vermutlich überwiegend die hanafitische Rechtsschule vorzufinden, welche hauptsächlich in der Türkei, Bosnien-Herzegowina, Afghanistan und Pakistan verbreitet ist (Lemmen 2001, S. 44). Die Schiiten in Deutschland stammen überwiegend aus dem Iran und aus Afghanistan, kleinere Gruppen auch aus dem Irak, der Türkei und dem Libanon (Wunn 2007, S. 123). Bereits ab den 1960er Jahren kamen zahlreiche Aleviten im Rahmen der Gastarbeitermigration und wenig später im Zuge der Familienzusammenführung nach Deutschland. In den 1980er Jahren folgten dann weitere als Asylsuchende, darunter zunehmend kurdische Aleviten aufgrund des Kurdenkonflikts in der Türkei. Die Anhänger der Ahmadiyya-Bewegung leben hauptsächlich auf dem indischen Subkontinent, daneben auch in Indonesien und West- und Ostafrika. Ihnen wird in Deutschland aufgrund von Verfolgung häufig Asyl gewährt.

21 Siehe Hunger 2005. 
ganisationen im Besonderen ${ }^{22}$ unternommen worden, teils beschränkt auf einzelne Bundesländer. ${ }^{23}$ Auch liegen inzwischen in Form von Fallstudien, die in unterschiedlichen Forschungskontexten entstanden sind, detaillierte Analysen zu einzelnen muslimischen Organisationen vor. ${ }^{24}$ Der bisherige Kenntnisstand deutet darauf hin, dass der organisierte Islam in Deutschland stark fragmentiert ist, nicht zu reden von denjenigen Muslimen, die von den Organisationen überhaupt nicht erreicht werden. So ergab die Studie von Riem Spielhaus aus dem Jahr 2006, dass rund die Hälfte der Berliner Moscheegemeinden von den größeren Verbänden unabhängig ist. ${ }^{25}$ Volkhard Krech kommt 2008 mit Blick auf das Land NRW zu einem ähnlichen Schluss. Laut seiner Erhebung organisieren die verbandsunabhängigen Gemeinden die meisten Muslime nach der DITIB ${ }^{26}$ (Diyanet İşleri Türk İslam Birliği - TürkischIslamische Union der Anstalt für Religion). ${ }^{27}$ Nach der Befragung des Bundesamts für Migration und Flüchtlinge (BAMF) aus dem Jahr $2008^{28}$ fühlten sich demgegenüber nur rund ein Viertel der Muslime von den im Koordinierungsrat der Muslime $(\mathrm{KRM})^{29}$ zusammengeschlossenen Verbänden Islamrat, Zentralrat der Musli-

22 Im Jahr 2009 führte das Zentrum für Türkeistudien und Integrationsforschung eine Bestandsaufnahme der muslimischen Organisationen in Deutschland durch, gefördert durch den EIF. Die entsprechende Datenbank ist Grundlage der Befragung in der hier vorgestellten Studie.

23 Siehe Spielhaus 2006; Hero et al. 2008 bzw., beruhend auf demselben Projekt, Chbib 2011.

24 Siehe Sökefeld 2008 b; Schiffauer 2010; Rosenow 2010; Jonker 2002.

25 Vgl. Spielhaus 2006, S. 15.

26 Als größter Verband muslimischer Gemeinden in Deutschland wurde die DITIB auf Initiative des türkischen Präsidiums für Religionsangelegenheiten mit dem Ziel gegründet, in Deutschland für die Migranten aus der Türkei einen laizistisch geprägten Islam zu etablieren. Bis heute ist die DITIB eng mit dem Amt für religiöse Angelegenheiten der Türkei verbunden und unterliegt dessen Kontrolle. Sie dürfte die (relative) Mehrheit der sunnitischen Muslime in Deutschland organisieren. Das Amt für Religionsangelegenheiten der Türkei übernimmt unter anderem die Besoldung und Ausbildung der Imame. Der Verband erhob lange Jahre den Anspruch, die Muslime in Deutschland allein zu vertreten. Vgl. auch Wunn 2007.

27 Siehe auch Chbib 2011.

28 Haug et al. 2009, S. 179.

29 Der KRM hat sich im Zuge der Deutschen Islamkonferenz konstituiert und ist als Reaktion der Verbände auf die Forderung nach einem einheitlichen Ansprechpartner für den deutschen Staat als Voraussetzung für die gesellschaftliche Integration zu verstehen. 
me (ZMD), DITIB und Verband der Islamischen Kulturzentren (VIKZ) ${ }^{30}$ vertreten. Dies ist zunächst kein Widerspruch, sagt die Zahl der Organisationen doch nichts über die individuellen Mitglieder aus. Auch ist davon auszugehen, dass eine beträchtliche Zahl von Menschen mit muslimischer Identität gar nicht organisiert ist, was zwangsläufig bei der Betrachtung ausschließlich der Organisationen für die Verbände ein günstigeres Bild ergibt - ihre Bedeutung ist bezogen auf den organisierten Islam größer als bezogen auf den Islam insgesamt. Die Studie des BAMF war insofern von unmittelbarer politischer Bedeutung, als sie als Begründung für die Teilnehmerauswahl der zweiten Auflage der DIK herangezogen wurde, indem das Verhältnis von verbandsangehörigen und muslimischen Einzelpersonen mit 1 zu 2 festgelegt wurde. ${ }^{31}$ Dies beinhaltet die politische Setzung, dass die Konferenz sich auf Menschen muslimischer Prägung bezieht, ungeachtet des religiösen Organisationsgrades oder der Gläubigkeit. ${ }^{32}$

Jenseits der Frage nach der Repräsentation gilt es, den Blick auf weitergehende Funktionen der muslimischen Organisationen und Verbände zu richten, speziell darauf, was die Mitgliedschaft oder Nichtmitgliedschaft in einem (bestimmten) Verband für die Organisationsstrukturen und Angebote der Gemeinden bedeutet hierüber gibt unsere Studie erstmals Auskunft. Auch diese Perspektive ist unerlässlich für eine Einschätzung der Bedeutung der Verbände für die Integration des Islams und der Muslime, ebenso wie die Repräsentationsthematik.

\subsection{Folgen intergenerationalen Wandels für die Organisationslandschaft}

Unabhängig von der aktuellen Bedeutung einzelner muslimischer Organisationen wird mit dem Heranwachsen der Nachfolgegenerationen die Erwartung an die Organisationen Veränderungen unterworfen sein, und die Konstellation, innerhalb

30 Mitglieder des Islamrats sind Verbände sowie regionale und lokale Vereinigungen, dominiert wird er von der IGMG. Die IGMG ist der größte (sunnitische) staatsunabhängige Verband, unterhielt aber in den 1980er Jahren enge Beziehungen zur islamistischen türkischen Wohlfahrtspartei. Der ZMD organisiert Verbände und Einzelorganisationen unterschiedlicher Herkunftsgruppen und Glaubensrichtungen. Der Verband der Islamischen Kulturzentren vertritt einen mystisch geprägten sunnitischen Islam, allerdings mit starker Orientierung auf Deutschland. Er war der erste Verband, der sich - schon in den 1960er Jahren - für die Schaffung einer gemeinsamen Bewegung auf Bundesebene einsetzte. Er stellte schon 1979 den Antrag auf Anerkennung als Körperschaft des öffentlichen Rechts und betreibt in Deutschland zahlreiche Koranschulen für Kinder und Jugendliche. Die bedeutendste alevitische Organisation ist die Alevitische Gemeinde Deutschland (AABF). Ihre Hauptziele sind die Etablierung des Alevitentums als eigenständige Glaubensrichtung und die Förderung der Rückbesinnung auf die alevitische Religion unter Jugendlichen. Siehe zu einer Übersicht und einer Charakterisierung der muslimischen Verbände in Deutschland Wunn 2007.

31 Siehe zur Zusammensetzung der Islamkonferenz und der Begründung dieser Zusammensetzung die Internetseite der DIK (www.deutsche-islam-konferenz.de, Zugriff vom 03.01.2012).

32 Vgl. Azzaoui 2011, S. 260. 
derer die Organisationen ihre Legitimität behaupten müssen, wird sich ändern. ${ }^{33}$ Es geht also nicht nur darum, welche Beiträge die muslimischen Gemeinden zur Sozialintegration leisten (können), sondern auch darum, wie die fortschreitende Sozialintegration von Muslimen in Deutschland auf die Arbeit der Gemeinden rückwirkt und von welcher Organisationslandschaft Integrationspolitik langfristig ausgehen kann.

Der aktuelle wissenschaftliche Kenntnisstand enthält Hinweise darauf, dass die etablierten, oft in Dachorganisationen oder Verbänden zusammengeschlossenen muslimischen Gemeinschaften in Deutschland durch intergenerationalen Wandel und Assimilation ihrer Klientel an Bedeutung verlieren könnten. Neugründungen von Gemeinden finden vermehrt außerhalb der etablierten Verbandsstrukturen statt. ${ }^{34}$ Dies könnte daran liegen, dass - idealtypisch - die Wurzeln der etablierten Organisationen in den Herkunftsländern sind und sie von Beginn an Menschen organisiert haben, die in ihrer Identität ebenfalls noch stark durch die Herkunftsländer geprägt waren. In den Nachfolgegenerationen bleibt nun möglicherweise zwar die religiös-kulturelle, nicht aber die geografische Herkunft von Bedeutung, ${ }^{35}$ da sich neue Identitäten herausbilden, die auch durch den Migrationshintergrund an sich bestimmt werden. ${ }^{36}$ Die deutsche Sprache gewinnt dann in Organisationen an Bedeutung und ermöglicht herkunftslandübergreifende Vergemeinschaftung. Unabhängig von dieser These haben intergenerationaler Wandel und der damit einhergehende Wertewandel Folgen für Organisationen, wenn sie Anhänger mobilisieren wollen.

Allerdings zeigen jüngere Fallstudien zu muslimischen Verbänden in Deutschland auch eine beträchtliche Dynamik dieser Organisationen. Sie passen sich mitunter in hohem Maße wechselnden Erwartungen ihres Umfelds wie auch den Bedürfnissen ihrer Klientel an. Im Falle von DITIB erkennt Rosenow eine weitgehende Identität der Erwartungen speziell deutscher integrationspolitischer Akteure und einer immer stärker in Deutschland verwurzelten Klientel, die zu vermehrter Zweisprachigkeit in der Arbeit, Differenzierung der Organisationsstrukturen, Professionalisierung und verstärktem sozialintegrativen Engagement geführt hat. Dessen ungeachtet verbleibt zwischen Deutschland- und Türkeiorientierung ein Spannungsfeld, das entsprechende Loyalitätskonflikte erzeugt. ${ }^{37}$

Anders als bei der in großem Umfang vom türkischen Staat abhängigen DITIB stellt sich der Fall der IGMG dar. Hier begünstigte der Niedergang der islamistischen Erbakan-Bewegung in der Türkei in den 1990er Jahren, deren europäischer Zweig die IGMG war, eine Verschiebung der Orientierung auf die europäischen Aufnah-

33 Vgl. Child et al. 1973.

34 Spielhaus 2006.

35 Dies kommt primär in der Übernahme der deutschen Staatsangehörigkeit zum Ausdruck; siehe zum Einbürgerungsgeschehen in Deutschland ausführlich Diehl, Blohm 2008.

36 Vgl. Tietze 2001, S. 237.

37 Rosenow 2010, S. 196-197.

Leviathan, 40. Jg., 1/2012 
megesellschaften ${ }^{38}$ und das »Empowerment ", die gesellschaftliche Stärkung der Muslime in Europa, bei Betonung der Bildungsbeteiligung und der religiösen Identität.

Die erwähnten Fallstudien machen deutlich, dass von einem Zusammenhang von Sozialintegration der Klientel, intergenerationalem Wandel und grenzüberschreitenden Kontakten der Gemeinden auszugehen ist, wobei aber Unklarheit über die genaue Ausgestaltung dieses Zusammenhangs besteht. ${ }^{39}$ Zum einen ist die assimilationstheoretisch anzunehmende Korrelation von automatisch fortschreitender Sozialintegration und stärkerer Aufnahmelandorientierung fraglich: Neuere internationale Befunde weisen darauf hin, dass gerade eine verbesserte Sozialintegration erst die Voraussetzung für transnationale Aktivitäten sein kann, was mit den jeweils zur Verfügung stehenden Ressourcen für die Pflege grenzüberschreitender Kontakte seitens der Individuen und Organisationen erklärt wird. ${ }^{40}$ Andererseits ist organisationaler Wandel nicht gleichbedeutend mit sozialem Wandel der Klientel. Und schließlich können grenzüberschreitende Netzwerke unterschiedliche Qualität haben: Der Islam in Europa kann einerseits vor dem Hintergrund der Arbeitsmigration im Sinne eines Zentrum-Peripherie-Modells interpretiert werden. Andererseits bildet der Islam in der Migration womöglich innerperiphere, europäische Verbindungen heraus, ${ }^{41}$ die bislang freilich empirisch nur wenig belegt sind. ${ }^{42}$ Ihnen kommt in normativer Hinsicht möglicherweise eine große Bedeutung zu: Im Zuge der zunehmenden Auseinandersetzungen mit dem Islam nach dem 11. September 2001

38 Halm et al. 2012.

39 In der bisherigen Forschung ist die Frage der transnationalen Orientierung von Migrantenorganisationen und ihres Zusammenhangs mit der Sozialintegration deutlich unterbelichtet geblieben. Wichtige Beiträge im Umfeld der Thematik: Koopmans thematisierte 2005 den Zusammenhang von Integrationspolitik und Herkunftslandorientierung von Migrantenorganisationen. Amelina und Faist verharren in ihrer Studie von 2008 auf einer diskursanalytischen Ebene, das heißt sie betrachten, wie die Organisationen den $\mathrm{Zu}$ sammenhang von Transnationalismus und Integrationsorientierung diskursiv verfertigen, nicht, wie sich dieser Zusammenhang tatsächlich darstellt. Nielsen leistete 2003 einen ersten Überblick über die Entwicklung transeuropäischer muslimischer Netzwerke, ohne allerdings systematische Bezüge zur Sozialintegration der Muslime herzustellen. Karam beleuchtete 2004 die Transnationalität im Kontext des politischen Islams, im Fokus der Aufmerksamkeit steht jedoch nicht das organisierte religiöse Leben der muslimischen Migranten in Europa in Gänze. Becker behandelte 2004 die wirtschaftlichen Potenziale des transnational organisierten muslimischen Geschäftslebens. Klause setzte sich 2006 im Rahmen seiner Studie zur Infrastruktur der Muslime in einer europäischen Grenzregion unter anderem mit der Frage der grenzüberschreitenden Kooperationen auseinander.

40 Siehe Portes et al. 2003; Portes et al. 2007.

41 Allievi 2003, S. 2.

42 Siehe die Befunde von Klause 2006, S. 145. Einen Hinweis auf eine solche Entwicklung birgt die Etablierung des Alevitentums in Europa, das, im Herkunftsland Türkei über Jahrzehnte unterdrückt und deshalb ohne Organisationsbeziehungen ins Herkunftsland, gleichermaßen eine europäische Transnationalität wie auch eine starke (auf den Nationalstaat gerichtete) Integrationsorientierung herausgebildet hat (Sökefeld 2008 a). 
wurden Rufe nach der Etablierung eines europaweiten Diaspora-Islam laut, der solche Netzwerke wohl voraussetzen würde. Von diesem versprach man sich auch Rückwirkungen auf Reformprozesse in der islamischen Welt. ${ }^{43}$ Solche politischen Hoffnungen widersprechen dann allerdings den Forderungen nach Assimilation und der Erwartung an die Aufgabe von Herkunftsland- zugunsten von Aufnahmelandnetzwerken.

Mit den vorliegenden Daten ist es nun möglich, systematisch vergleichend darzustellen, wie sich der intergenerationale Wandel in unterschiedlichen Gemeinden manifestiert und auf die (auch Landesgrenzen überschreitende) Tätigkeit auswirkt.

\section{Vorgehensweise}

Um einen Beitrag zur Beantwortung der oben aufgeworfenen Fragen zu leisten, führte die Stiftung Zentrum für Türkeistudien und Integrationsforschung (ZfTI) im ersten Halbjahr 2011 eine telefonische, computergestützte standardisierte Befragung von Moscheegemeinden durch. Erkenntnisgegenstand waren die muslimischen einschließlich der alevitischen ${ }^{44}$ Gemeinden in Deutschland, wobei wir, unabhängig von der rechtlichen Organisationsform, unter einer Gemeinde diejenigen Organisationen verstehen, die über Räumlichkeiten für religiöse Veranstaltungen verfügen.

\subsection{Fragebogenentwicklung}

Es war aufgrund der unterschiedlichen Glaubenspraxis notwendig, eine teilweise abweichende Variante des Fragebogens für die alevitischen Gemeinden zu erstellen, was speziell die Erhebung grundsätzlicher Strukturmerkmale der Organisationen betraf (religiöse Angebote und ihre Frequenz usw.). ${ }^{45}$ Der Fragebogen wurde mit den Verbänden diskutiert und abgestimmt und in deutscher, türkischer und in arabischer Sprache erstellt, da die Ansprache in der Muttersprache die Teilnahmebereitschaft erheblich erhöht. ${ }^{46}$ Die elf Interviewer (Muttersprachler) führten nach der Besprechung des Fragebogens und der besonderen Gesprächssituationen einige Test-Interviews durch.

43 So der damalige Bundestagspräsident Wolfgang Thierse in der Rheinischen Post vom 27.12.2001.

44 Es ist umstritten, ob die Aleviten zur muslimischen Gemeinschaft zu rechnen sind. Die Föderation der Aleviten-Gemeinden in Europa (AABF) sieht das Alevitentum als eigenständige Religion und nicht als eine Glaubensrichtung innerhalb des Islams an. Unterschiede zu Schiiten und Sunniten bestehen in der theologischen Vorstellung sowie in der Glaubensausübung. Dem Imam im sunnitischen Islam steht bei den Aleviten der Dede gegenüber, der Moschee das Cem-Haus und dem gemeinsamen Gebet die Cem-Feier und rituelle Musik bzw. ritueller Tanz.

45 Da sich die Frequenz und Regelmäßigkeit solcher Aktivitäten zwischen den Glaubensrichtungen stark unterscheiden, sind sie nur sehr bedingt geeignet, zum Vergleich etwa des Aktivitätsgrads der Gemeinden herangezogen zu werden.

46 Blohm, Diehl 2001.

Leviathan, 40. Jg., 1/2012 


\subsection{Grundgesamtheit, Durchführung und Ausschöpfung}

Grundlage der Befragung war eine Datenbank, die das ZfTI im Jahr 2009 erarbeitet hatte. Nach Ergänzungen durch das BAMF verzeichnete die Datenbank 2.804 Organisationen mit Telefonnummern zuzüglich 496 Organisationen ohne Telefonnummern.

Die Befragung wurde von Februar bis Juni 2011 wochentags zwischen 13.00 und 18.00 Uhr sowie samstags zwischen 11.00 Uhr und 17.00 Uhr durchgeführt und erfolgte in 20 Wellen. Zusätzlich wurden diejenigen Gemeinden, für die keine Telefonnummern vorlagen, brieflich kontaktiert.

Insgesamt wurden 1.141 Interviews durchgeführt, 1.113 davon telefonisch und 28 postalisch. Dies entspricht einer Ausschöpfungsquote von $35 \%$, legt man die ursprüngliche Zahl der aufgelisteten Gemeinden zugrunde. Bezogen auf die in den 20 Befragungswellen erreichten Anschlüsse liegt die Ausschöpfungsquote bei $63 \%$. In der Ausgangsliste erwiesen sich $22 \%$ der Telefonnummern als falsch. Aufgrund des Rücklaufs von telefonischer und schriftlicher Befragung wurde die Adressdatenbank bereinigt. Im Ergebnis ist damit von 2.342 Gemeinden mit Gebetsräumlichkeiten im Befragungszeitraum auszugehen.

Die Interviews erfolgten mit Gesprächspartnern in unterschiedlichen Funktionen - zu $33 \%$ mit den Gemeindevorsitzenden, $23 \%$ mit Vorstandsmitgliedern, $21 \%$ mit den Imamen bzw. Dedes, $15 \%$ mit Gemeindemitgliedern und $4 \%$ mit Angestellten. Die weiteren Interviews wurden mit sonstigen Personen geführt (3\% insgesamt).

\subsection{Verhältnis der Vergleichsmerkmale in Interviews und Ausgangsadressliste}

Die Studie hat insofern keinen repräsentativen Charakter, als Repräsentativität nicht nur die Bekanntheit der Grundgesamtheit voraussetzt, sondern auch eine zufällige Stichprobenziehung. Beide Bedingungen sind hier nicht erfüllt. Ein Vergleich der bereinigten Datenbank mit den Ergebnissen der Befragung zeigt indessen hinsichtlich der verfügbaren Vergleichsmerkmale Glaubensrichtung und Verbandszugehörigkeit, dass die Befragtengruppe die (bereinigte) Ausgangsliste im Wesentlichen widerspiegelt. Der Anteil der alevitischen Gemeinden ist mit je 4,7\% sogar exakt identisch. Große Unterschiede zeigen sich nur zwischen dem großen Anteil von Gemeinden, bei denen die Glaubensrichtung in der Ausgangsdatenbank (noch) nicht bekannt war $(20 \%)$, und dem geringeren diesbezüglichen Anteil in der Befragung $(1 \%)$. Geht man plausiblerweise davon aus, dass der Großteil dieser Gemeinden sunnitisch geprägt ist, kommt der Anteil von $83 \%$ sunnitischer Gemeinden in der Befragung den Ausgangsdaten ebenfalls nahe. 
Tabelle 1: Vergleich der Glaubensrichtung in bereinigter Datenbank und Befragung

\begin{tabular}{|l|r|r|r|r|}
\hline & $\begin{array}{c}\text { Absolut in } \\
\text { Datenbank }\end{array}$ & $\begin{array}{c}\text { Anteil an Ein- } \\
\text { trägen in Daten- } \\
\text { bank } \\
\text { (in \%) }\end{array}$ & $\begin{array}{c}\text { Absolut Nen- } \\
\text { nungen in Be- } \\
\text { fragung }\end{array}$ & $\begin{array}{c}\text { Anteil an Nennun- } \\
\text { gen in Befragung } \\
\text { (in \%) }\end{array}$ \\
\hline Sunnitisch & 1.573 & 67,2 & 951 & 83,3 \\
\hline Schiitisch & 61 & 2,6 & 25 & 2,2 \\
\hline Ahmadi & 71 & 3,0 & 21 & 1,8 \\
\hline Sufi & 13 & 0,6 & 4 & 0,4 \\
\hline Gemischt & 35 & 1,5 & 73 & 6,4 \\
\hline Alevitisch & 111 & 4,7 & 54 & - \\
\hline Sonstige & 4 & 0,2 & 13 & 1,1 \\
\hline Weiß nicht/k.A. & 474 & 20,2 & 1.141 & 100 \\
\hline Total & 2.342 & 100 & -7 & - \\
\hline
\end{tabular}

Dasselbe gilt für die Verbandszugehörigkeit. Vergleicht man nur diejenigen Gemeinden, für die in der bereinigten Datenbank ein Eintrag über eine Mitgliedschaft vorliegt, mit den angegebenen Verbandsmitgliedschaften in der Befragung, so sind die Anteile der großen Verbände ähnlich hoch, mit Ausnahme der IGMG, die in der Befragung deutlich überrepräsentiert ist. Sowohl die bereinigte Datenbank als auch die Befragungsdaten beinhalten Doppelmitgliedschaften, die sich im ersten Fall (selten, 22-mal) aus der gleichzeitigen Mitgliedschaft in Verbänden und regionalen $\mathrm{Zu}$ sammenschlüssen ergeben, während in der Befragung noch gleichzeitige Mitgliedschaften in mehreren Verbänden hinzukommen (195 Organisationen mit Mehrfachmitgliedschaft insgesamt). Hierin liegt eine Unschärfe der Daten begründet. ${ }^{47}$

Während die Anteile der Nennungen der Verbände bzw. der Einträge sich ungefähr entsprechen, gilt dies nicht mit Blick auf die Zugehörigkeit der jeweiligen Gemeinden. Sind in der bereinigten Datenbank 853 Gemeinden ohne jede Verbandszugehörigkeit verzeichnet (36\%), so sind dies unter den schließlich befragten Ge-

47 Speziell die Mehrfachmitgliedschaft in großen Verbänden, etwa in DITIB und IGMG, scheint nicht unbedingt plausibel, ist aber nicht ausgeschlossen. Möglicherweise kommen hier unexakte Informationen zum Tragen, die von einem Verbandswechsel herrühren, möglicherweise auch andere Motive der Organisationen, in mehreren Verbänden Mitglied zu sein. Wir betrachten in den folgenden Auswertungen jeweils alle genannten Mitgliedschaften. Alternativ wurde bei allen Zusammenhängen mit der Mitgliedschaftsvariablen auch nur unter Einbezug der verbandsfreien und der nur in einem Verband organisierten Gemeinden gerechnet, wobei Signifikanzniveaus und Effektstärken zumeist nur gering variierten. Bei deutlich abweichenden Ergebnissen oder Konsequenzen für unsere Argumentation wird auch die alternative Berechnung dargestellt. 
meinden noch 278 (12\%). Dies mag größtenteils daran liegen, dass bisher unbekannte Verbandszugehörigkeiten im Rahmen der Befragung bekannt wurden. Es lässt aber auch das Problem erkennen, dass die Teilnahme an der Befragung möglicherweise von den Ressourcen der Gemeinde abhängt (Besetzung von Büros, Öffnungszeiten etc.), die wir anhand der vorhandenen Daten zur Grundgesamtheit nicht kontrollieren können. Nicht auszuschließen ist, dass große und ressourcenstarke Gemeinden in unserer Befragung überrepräsentiert sind (und damit auch verbandszugehörige Gemeinden). Die Durchführung von 20 Befragungswellen sollte dieses Problem weitgehend minimiert haben, allerdings beträgt, bezogen auf die bereinigte Ausgangsdatenbank, die Ausschöpfungsquote der dort ohne Verbandszugehörigkeit verzeichneten Gemeinden nur 35 \% gegenüber 49 \% insgesamt. Trotz der genannten Beschränkungen sollten die Ergebnisse aber aussagekräftig für die Landschaft der Moscheegemeinden in Deutschland sein. Der Text trägt der Restunsicherheit über das Verhältnis von befragten Gemeinden und Grundgesamtheit dennoch Rechnung, indem er hauptsächlich auf Zusammenhänge zwischen Merkmalen der Gemeinden abstellt, weniger auf die Deskription der »realen « Organisationslandschaft.

Tabelle 2: Vergleich der Verbandszugehörigkeit in bereinigter Datenbank und Befragung 48

\begin{tabular}{|l|l|l|l|l|}
\hline & \multicolumn{1}{|c|}{$\begin{array}{c}\text { Absolut in } \\
\text { Datenbank }\end{array}$} & $\begin{array}{c}\text { Anteil an } \\
\text { Einträgen in } \\
\text { Datenbank } \\
\text { (in \%) }\end{array}$ & $\begin{array}{c}\text { Absolut Nennun- } \\
\text { gen in Befragung } \\
\text { (Mehrfachnen- } \\
\text { nungen möglich) }\end{array}$ & $\begin{array}{c}\text { Anteil an Nennun- } \\
\text { gen in Befragung } \\
\text { (in \% Mehrfach- } \\
\text { nennungen mög- } \\
\text { lich) }\end{array}$ \\
\hline AMJ & 33 & 2,2 & 11 & 1,0 \\
\hline ATIB & 22 & 1,5 & 18 & 1,6 \\
\hline DITIB & 717 & 48,0 & 514 & 45,0 \\
\hline IGBD & 40 & 2,7 & 16 & 1,4 \\
\hline IGD & 2 & 0,1 & 2 & 0,2 \\
\hline IGMG & 192 & 12,9 & 220 & 19,3 \\
\hline IGS & - & - & 3 & 0,3 \\
\hline
\end{tabular}

48 Abkürzungen: AMJ = Ahmadiyya Muslim Jamaat; ATIB = Union der Türkisch-Islamischen Kulturvereine in Europa; DITIB = Türkisch-Islamische Union der Anstalt für Religion; IGBD = Islamische Gemeinschaft der Bosniaken in Deutschland; IGD = Islamische Gemeinschaft in Deutschland; IGMG = Islamische Gemeinschaft Milli Görüş; IGS = Islamische Gemeinschaft der Schiitengemeinden Deutschlands; Türk Fed = Türkische Föderation; UIAZD = Union der Islamisch-Albanischen Kulturzentren; VIKZ = Verband der islamischen Kulturzentren; ZMD = Zentralrat der Muslime in Deutschland; AABF = Alevitische Gemeinde; KRM = Koordinierungsrat der Muslime; siehe zu einer Charakterisierung dieser Verbände Wunn 2007. 


\begin{tabular}{|l|l|l|l|l|}
\hline & \multicolumn{1}{|c|}{$\begin{array}{c}\text { Absolut in } \\
\text { Datenbank }\end{array}$} & $\begin{array}{c}\text { Anteil an } \\
\text { Einträgen in } \\
\text { Datenbank } \\
\text { (in \%) }\end{array}$ & $\begin{array}{c}\text { Absolut Nennun- } \\
\text { gen in Befragung } \\
\text { (Mehrfachnen- } \\
\text { nungen möglich) }\end{array}$ & $\begin{array}{c}\text { Anteil an Nennun- } \\
\text { gen in Befragung } \\
\text { in \% } \text { Mehrfach- } \\
\text { nennungen mög- } \\
\text { lich) }\end{array}$ \\
\hline Islamrat & 2 & 0,1 & 7 & 0,6 \\
\hline Jama at un Nur & 15 & 1,0 & 16 & 1,4 \\
\hline Türk Fed & 13 & 0,9 & 7 & 0,6 \\
\hline UIAZD & 13 & 0,9 & 7 & 0,6 \\
\hline VIKZ & 226 & 15,2 & 190 & 16,7 \\
\hline ZMD & 15 & 1,0 & 20 & 1,8 \\
\hline AABF & 87 & 5,8 & 42 & 3,7 \\
\hline $\begin{array}{l}\text { Unbestimmt, } \\
\text { sonstige }\end{array}$ & 57 & 3,8 & 33 & 2,9 \\
\hline $\begin{array}{l}\text { Schuras/Lan- } \\
\text { deszusammen- } \\
\text { schlüsse }\end{array}$ & 55 & 3,7 & 31 & 100 \\
\hline KRM & - & 100 & 140 & 2,7 \\
\hline $\begin{array}{l}\text { Einträge/Nen- } \\
\text { nungen gesamt }\end{array}$ & 1.489 & - & 3 & 100 \\
\hline
\end{tabular}

\section{Strukturen und Angebote der Gemeinden ${ }^{49}$}

\subsection{Weitere Grunddaten der befragten Gemeinden - Herkunft der Besucher, Organisationsalter, Rechtsform}

Die Moscheegemeinden sind in hohem Maße heterogen, wobei allerdings in einzelnen Gemeinden Gläubige aus der Türkei oft deutlich dominieren. Die Befragten in den Gemeinden wurden gebeten, die Herkunft der Moscheebesucher anzugeben, wobei Mehrfachnennungen (ohne Vorgaben) möglich waren. ${ }^{50}$ Die häufigste Nennung war erwartungsgemäß die Türkei (40 \% der Nennungen; 81 \% der Gemeinden werden - auch - von Gläubigen aus der Türkei besucht). ${ }^{51}$ Der Normalfall - mit rund zwei Dritteln - ist jedoch eine heterogene Zusammensetzung der Gemeinden.

49 Falls im Text nicht anders vermerkt, beziehen sich die Angaben hier und im Folgenden auf alle Gemeinden ( $\mathrm{N}=1.141)$.

50 Diese und die folgend dargestellten Herkunftsfragen wurden den Aleviten nicht gestellt, da hier per se von der türkischen Herkunft ausgegangen wurde. In der vorliegenden Auswertung wurden die 54 alevitischen Gemeinden zu den türkisch dominierten Organisationen hinzugerechnet, um sie in den folgenden, auf dieser Variablen beruhenden Berechnungen berücksichtigen zu können.

51 Ohne Berücksichtigung der Fälle, in denen keine Angaben gemacht wurden. 
So geben $43 \%$ an, von drei oder mehr Herkunftsgruppen besucht zu werden, zwei Herkunftsgruppen werden in $25 \%$ der Fälle genannt. Fragt man jedoch nach der jeweils dominierenden Herkunftsgruppe - in dem Sinne, dass diese über $70 \%$ der Besucher stellt -, so herrscht in lediglich $5 \%$ der Gemeinden keine bestimmte Gruppe vor. Die meisten $(64 \%)$ werden von Türkeistämmigen dominiert, mit weitem Abstand gefolgt von Bosniern (5\%) und Pakistanis (3\%). Alle anderen festgestellten Gruppen machen weniger als $1 \%$ aus. ${ }^{52}$ Hinzuweisen ist auf den hohen Anteil der Gemeinden, die auf diese Frage keine Antwort gaben (18\%). Insgesamt erscheinen die Moscheegemeinden in Deutschland zwar nach Herkunftsgruppen gemischt, zugleich aber auch - erwartungsgemäß - deutlich türkisch dominiert.

Die meisten der untersuchten Gemeinden wurden zwischen 1980 und 1999 gegründet, $34 \%$ in den 1980er Jahren und weitere $29 \%$ in den 1990er Jahren. Davor existierten nur $19 \%$, ab 2000 wurden lediglich weitere $13 \%$ gegründet. Dies weist darauf hin, dass sich die muslimische Organisationslandschaft in Deutschland weitgehend etabliert hat und trotz der großen Dynamik des Organisationsfelds der Großteil der Gemeinden schon länger als zehn Jahre besteht und im Zuge der Verfestigung der Aufenthalte und des Familiennachzugs entstanden ist.

Die Gemeinden sind zu $91 \%$ als eingetragene Vereine organisiert, 2 \% bezeichnen sich als Initiativen oder Netzwerke, $1 \%$ als sonstige Zusammenschlüsse.

\subsection{Größe, Ausstattung und weitere Ressourcen}

Die Frequenz der unterschiedlichen Angebote der Gemeinden wurde mit einer Reihe von Fragen erhoben, differenziert nach Frauen und Männern, aber auch nach religiösen und nichtreligiösen Angeboten (bei letzteren auch unterschieden nach Jugendlichen und Erwachsenen).

Die Teilnahme an religiösen Angeboten fokussierte auf den Besuch des Freitagsgebets bzw. bei den Aleviten allgemein - in Ermangelung eines Freitagsgebets - auf die üblichen Gemeindetreffen am Wochenende. Der Vergleich der alevitischen mit den übrigen Gemeinden zeigt für erstere eine annährende Gleichverteilung des Gemeindebesuchs am Wochenende zwischen Männern und Frauen, während beim Freitagsgebet die Frauen massiv unterrepräsentiert sind - bei $55 \%$ aller befragten Gemeinden kommen weniger als 20 Frauen und Mädchen zum Freitagsgebet. Eine Erklärung ist, dass das Freitagsgebet für Musliminnen keine religiöse Pflicht darstellt.

Der Besuch des Gebets ist nur bedingt geeignet, Aufschluss über die Gemeindegröße zu geben, einerseits aufgrund des Problems der Teilnahme von Frauen, andererseits, wie sich noch zeigen wird, weil die meisten Gemeinden auch zahlreiche nichtreligiöse Angebote machen. Als Hinweis auf die unterschiedlichen Gemeindegrößen ist vielmehr die Information geeignet, wie viele Menschen durch die Ange-

52 Aufgrund der nichtstandardisierten Antwortmöglichkeit wurden mitunter - in seltenen Fällen - nicht Länder, sondern Herkunftsregionen genannt, die keine genaue Zuordnung erlaubten, auch aufgrund zum Teil definitorischer Unklarheit (beispielsweise "Arabien «, »Naher Osten«). In der Studie wurden diese Nennungen (2\%) unter » andere/nicht zuzuordnen « zusammengefasst. 
bote der Gemeinden insgesamt erreicht werden. ${ }^{53}$ Die Größe der befragten Moscheegemeinden ist sehr unterschiedlich, mit $48 \%$ der Gemeinden, die bis 200 Gläubige erreichen, und $41 \%$, die ihre Klientel als noch größer einschätzen. Allerdings sind sehr kleine und kleinere Gemeinden (bis 20 Besucher bzw. 21-50 Besucher) mit einem Anteil von $4 \%$ bzw. $12 \%$ eher selten - die meisten Gemeinden erreichen über 50 Menschen.

Unter den befragten Gemeinden ist mit $98 \%$ die Verfügung über feste, eigene Gebetsräume der Regelfall. Fast bei allen nichtalevitischen Gemeinden (95\%) existieren abgetrennte Gebetsmöglichkeiten für Frauen, trotz ihrer starken Unterrepräsentation bei den Freitagsgebeten. Ebenfalls eine große Mehrheit der Gemeinden $(81 \%)$ verfügt über zusätzliche Räumlichkeiten für Feizeit- und Bildungsangebote. Nur eine Minderheit ist hingegen Eigentümerin der Räumlichkeiten (29\%), erzielt Mieteinnahmen (22\%) oder unterhält eine repräsentative Moschee mit Minarett (12\%, ohne Aleviten). Die befragten Gemeinden sind ganz überwiegend durchgängig zugänglich $-95 \%$ haben an vier oder mehr Tagen in der Woche geöffnet. $39 \%$ der Gemeinden planen Neubauten, so dass sich die räumliche Ausstattung zukünftig in beträchtlichem Umfang verbessern könnte.

Hinsichtlich der Organisationsstrukturen erscheinen die befragten Gemeinden mehrheitlich recht differenziert, was angesichts des grundsätzlich ehrenamtlichen Charakters der Tätigkeiten nicht überrascht. 93 \% der Gemeinden haben einen eigenen Imam bzw. Dede. Zuzüglich nur aushilfsweise in den Gemeinden tätiger Imame oder Dedes verfügen nahezu alle Gemeinden über einen Vorbeter. Aber auch andere, nichtreligiöse Aufgaben werden in den Gemeinden in der Mehrheit durch Beauftragte oder Abteilungen wahrgenommen: Über einen Kassenwart verfügen nach eigenen Angaben $90 \%$ der Gemeinden - angesichts der fast durchgängigen Verfasstheit als e.V. nicht überraschend -, aber auch Frauen- (84\%), Jugend(83 \%), Dialog- (79\%), Öffentlichkeitsarbeits- $(78 \%)$, Bildungs- $(74 \%)$ und Sportabteilungen $(52 \%)$ bzw. -beauftragte werden mehrheitlich genannt. Über gesonderte Abteilungen für Senioren verfügen immerhin $46 \%$ der Gemeinden, eine Gruppe, die angesichts der Migrationsgeschichte der Muslime in Deutschland erst in jüngerer Zeit an Bedeutung gewinnt.

Insgesamt lässt sich die Ressourcensituation der Gemeinden so charakterisieren, dass grundlegende Infrastrukturelemente wie ein Vorbeter und Räumlichkeiten durchgängig vorhanden sind und eine Aufgabendifferenzierung in den Gemeinden besteht. Eine größere Varianz gibt es demgegenüber bei Merkmalen, die Nachhaltigkeit indizieren: Immobilieneigentum und repräsentative Moscheebauten.

\subsection{Religiöse und nichtreligiöse Angebote}

Die Gemeinden machen zahlreiche religiöse Angebote über Gebete und die Feier von Gottesdiensten hinaus. Eine große Mehrheit der nichtalevitischen Organisationen bietet Korankurse bzw. Islamunterricht für Kinder und Jugendliche an (96 \%), von jeweils über $80 \%$ der Gemeinden werden interne Iftar-Essen (Fastenbrechen

53 Diese Variable ist dann auch Grundlage der weiteren Analysen.

Leviathan, 40. Jg., 1/2012 
nach Sonnenuntergang im Ramadan), Feiern der heiligen Nächte (Geburtstag des Propheten, Himmelsreise des Propheten etc.), Wallfahrten und Begräbnisfeiern ausgerichtet. Jeweils mehr als die Hälfte bieten Spendensammlungen (Zekat), Feiern anlässlich von Beschneidung und Eheschließung und die Vermittlung von Tieropfern anlässlich des Opferfestes an. Die alevitischen Gemeinden wurden nur nach der Existenz von Saz- und Semah-Kursen gefragt. Auch hier ist das Angebot solcher Kurse mit $93 \%$ bzw. $91 \%$ die Regel.

Die meisten Gemeinden machen weit über religiöse Dienstleistungen hinausgehende Angebote. Diese Entwicklung hat sich in der Konsolidierungsphase der Organisationen in den letzten Jahren verstärkt, zum einen aufgrund integrationspolitischer Erwartungen des Aufnahmelandes, zum anderen aber auch wegen des intergenerationalen Wandels der Klientel. ${ }^{54}$ Diese Angebote wurden für Jugendliche und Erwachsene getrennt erfragt, weil angenommen wurde, dass sich die Angebotsstrukturen für diese Gruppen unterscheiden. Die Ergebnisse bestätigen diese Vermutung. So sind etwa Sportangebote für Jugendliche sehr viel häufiger als für Erwachsene. Bestimmte Angebote sind zudem von vornherein nur für eine der beiden Gruppen von Bedeutung (wie etwa Hausaufgabenhilfe).

Bemerkenswert ist, dass deutsche Sprachkurse sowohl für Jugendliche als auch für Erwachsene deutlich häufiger angeboten werden als Kurse der Herkunftssprachen. Immerhin ein knappes Drittel der Gemeinden bietet deutsche Sprachkurse für Jugendliche an. Überhaupt dominieren keineswegs Herkunftskultur und Traditionspflege die nichtreligiöse Tätigkeit der Gemeinden, im Gegenteil nehmen Orientierungshilfen für die deutsche Gesellschaft (Sozial-, Erziehungs- und Gesundheitsberatung, Hausaufgabenhilfe, interreligiöser Dialog) einen breiten Raum ein.

Tabelle 3: Nichtreligiöse Angebote der Gemeinden (in \% aller befragten Gemeinden, Mehrfachnennungen)

\begin{tabular}{|l|r|r|}
\hline & für Jugendliche & für Erwachsene \\
\hline Sport/Bewegung & 72,2 & 25,6 \\
\hline Gesellschaftskunde/Exkursionen & 66,5 & 48,2 \\
\hline Interreligiöser Dialog & 65,2 & 60,4 \\
\hline Hausaufgabenhilfe & 57,3 & $-*$ \\
\hline Sprachkurse Deutsch & 31,0 & 23,5 \\
\hline Musik, Kultur, Tanz, Folklore & 31,0 & 11,1 \\
\hline Sprachkurse Herkunftssprache & 29,8 & 12,4 \\
\hline Computer, EDV & 27,7 & 15,2 \\
\hline Handarbeiten, Basteln, Kochen & 19,2 & 14,6 \\
\hline
\end{tabular}

54 Vgl. Rosenow 2010, S. 177. 


\begin{tabular}{|l|r|r|}
\hline & für Jugendliche & \multicolumn{1}{|c|}{ für Erwachsene } \\
\hline Integrationskurse (verpflichtend lt. Gesetz) & $-*$ & $20,2^{55}$ \\
\hline Einzelhandel, Friseur etc. & $-*$ & 10,3 \\
\hline Teestube & $-*$ & 83,8 \\
\hline Gesundheitsberatung & $-*$ & 36,5 \\
\hline Erziehungsberatung & $-*$ & 43,0 \\
\hline Sozialberatung & $-*$ & 43,2 \\
\hline Sonstiges & 3,2 & 1,8 \\
\hline Keine Angebote & 6,1 & 1,7 \\
\hline
\end{tabular}

* nicht abgefragt

\section{Integrationsbeiträge, sozialer Wandel und Organisationsstrukturen}

\subsection{Integrationsbeiträge - Offenbeit und Vielfalt der Angebote}

Eingangs haben wir bereits problematisiert, wie schwierig die Messung von »Integrationsbeiträgen « der Gemeinden ist. Es gilt, sowohl die Binnenwirkungen in der Migrationsbevölkerung als auch den Einschluss in die Aufnahmegesellschaft als Potenziale der Sozialintegration zu berücksichtigen. Die Existenz von Angeboten für unterschiedliche soziale Gruppen (Frauen, Senioren, Neuzuwanderer etc.) hat nach unserer Ansicht eine die Sozialintegration begünstigende Wirkung, da unterstellt werden kann, dass Ausgangspunkt der Angebote wenigstens zum Teil die Spezifik der Lebenssituation der Zielgruppen in Deutschland ist und dadurch die Vergesellschaftung für Gruppen mit ansonsten schwierigen Kontaktvoraussetzungen erleichtert wird. Soweit erkennbar, hat der Einbezug von Nichtmuslimen oder Menschen ohne Kenntnis der Herkunftssprachen in die Arbeit der Gemeinden ebenfalls eine günstige Wirkung auf die Sozialintegration, weil Netzwerke der Individuen sich ausdifferenzieren und interkulturellen Konflikten vorgebeugt wird. ${ }^{56}$ Hier spielt dann auch eine Rolle, in welcher Sprache religiöse Veranstaltungen in den Gemeinden durchgeführt werden. Untersucht wurde darüber hinaus, in welchem Umfang von der Aufnahmegesellschaft finanzierte und (normativ) als sozialintegrativ wirksam definierte Maßnahmen durchgeführt werden.

55 Dieser Anteil erscheint hoch, auch unter Einbezug der Möglichkeit einer Überrepräsentation angebotsstarker Gemeinden in der Befragung. Es ist davon auszugehen, dass es sich nicht in jedem Fall tatsächlich um Integrationskurse lt. Zuwanderungsrecht handelt, sondern auch um nicht anerkannte Angebote. Hierfür spricht, dass nur $15 \%$ der Gemeinden angeben, überhaupt schon einmal von deutschen Stellen finanzierte Projekte durchgeführt zu haben. Bei letzterer Frage geben nur 20 Gemeinden die Durchführung von Integrationskursen an, was einem Anteil von $2 \%$ entsprechen würde.

56 Die vermutliche Freiwilligkeit solcher Kontakte spricht für diese Lesart; vgl. Pettigrew, Tropp 1998. 
$15 \%$ der Gemeinden haben bereits Integrationsprojekte oder Ähnliches durchgeführt, die von deutschen Stellen finanziert wurden. Unter den Nennungen dominieren deutsche Sprachkurse, Begegnungsveranstaltungen und Integrationskurse. Vertreten sind ebenso Qualifizierungsmaßnahmen, häufig für Jugendliche. Unter den nichtalevitischen Gemeinden geben $83 \%$ an, bereits einmal am »Tag der offenen Moschee" teilgenommen zu haben, was auf eine große Öffnungsbereitschaft der Moscheen zur Aufnahmegesellschaft hindeutet. Fragt man aber danach, in welchem Umfang Deutsche an den nichtreligiösen Angeboten der Gemeinden teilnehmen, so zeigen sich nur bedingt Verbindungen. An den nichtreligiösen Angeboten für Jugendliche bzw. für Erwachsene nehmen zu 48 \% bzw. zu 43 \% vereinzelt auch Deutsche teil. ${ }^{57}$ In größerem Umfang beteiligen sich allerdings bei den Jugendangeboten nur zu $4 \%$ und bei den Erwachsenenangeboten zu $5 \%$ Deutsche. Alle Angebote und Antwortalternativen zusammengenommen, ergibt sich immerhin ein Anteil von $59 \%$ der Gemeinden, an deren nichtreligiösen Angeboten zumindest vereinzelt Deutsche teilnehmen. ${ }^{58}$

Gefragt wurde auch nach dem Anteil von Mädchen und Frauen an den nichtreligiösen Angeboten der Gemeinden. 65 \% der Gemeinden mit Angeboten für Jugendliche bieten gemeinsame Aktivitäten für Jungen und Mädchen an. $76 \%$ haben spezielle Angebote für Mädchen, ${ }^{59} 58 \%$ aller Gemeinden solche für Frauen. Diese Voraussetzungen führen zu einem relativ hohen Anteil von Mädchen und Frauen unter den Nutzern nichtreligiöser Angebote, wobei aber dennoch eine Unterrepräsentation des weiblichen Geschlechts zu konstatieren bleibt. Dabei ist die Situation bei Mädchen und Frauen sehr ähnlich. Zu jeweils $67 \%$ bzw. $63 \%$ geben die Gemeinden an, dass bis zu $50 \%$ derjenigen, die die Angebote wahrnehmen, weiblichen Geschlechts sind, ${ }^{60}$ nur $20 \%$ bzw. $19 \%$ der Befragten in den Gemeinden beobachten eine weibliche Teilnahme von über $50 \%$.Viele Gemeinden betreiben außerdem weitere spezielle Zielgruppenaktivitäten. $37 \%$ haben Angebote für islaminteressierte Nichtmuslime, $30 \%$ Angebote für Senioren, und $17 \%$ richten sich an Neueinwanderer.

Das religiöse Angebot der Gemeinden ist zumeist in der Herkunftssprache gehalten (zu $85 \%$, Mehrfachnennungen möglich), zu immerhin $37 \%$ findet es aber auch in deutscher Sprache statt, und zu $25 \%$ wird es ins Deutsche übersetzt. Übersetzungen in die Herkunftssprache sind mit $3 \%$ selten. Dieser Befund deutet auf einen Wandel zur deutschen Sprache in den befragten Gemeinden hin, der im Zusammenhang mit einer Bedeutungsminderung der Herkunft im intergenerationalen Wandel zu tun haben dürfte. Offenbar sind zahlreiche Gemeinden in der Lage, auf diesen Wandel zu reagieren.

Die bisher vorgestellten Befunde zeigen, dass die Gemeinden bezüglich ihrer Angebote und deren Offenheit gegenüber unterschiedlichen Gruppen deutlich variie-

$57 \mathrm{~N}=1.071$ bzw. 1.076 .

$58 \mathrm{~N}=1.121$.

$59 \mathrm{~N}=1.071$.

$60 \mathrm{~N}=1.071$ bzw. 1.076 . 
ren. Es stellt sich daher die Frage, womit sich das erklären lässt. Wir greifen zurück auf unsere Vorüberlegungen: Der Forschungsstand lässt hier nur bedingt die Ableitung von Annahmen zu, welche Merkmale die Aufnahmelandorientierung der Gemeinden beeinflussen. Allerdings könnten wir im politischen Diskurs vorherrschende Vorstellungen prüfen, beispielsweise die Frage, ob die Gruppe der Aleviten tatsächlich weniger segregationsorientiert ist als sunnitische oder schiitische Muslime. Doch selbst wenn dem so wäre, so sagt dies wenig über das Organisationshandeln aus, das zusätzlichen Einflussfaktoren unterliegt. Daher ist kaum zu erwarten, dass sich solche Zusammenhänge in unserer Datenanalyse zeigen. Am nächsten liegt noch die Annahme, dass diejenigen Moscheen, in denen keine bestimmte Herkunftsgruppe dominiert, möglicherweise in größerem Umfang aufnahmelandbezogene Angebote machen als Gemeinden mit dominierenden Herkunftsgruppen. Umstritten ist dabei freilich, ob ein Konkurrenzverhältnis zwischen Aufnahme- und Herkunftslandorientierung besteht. Von entscheidender Bedeutung für die Aufnahmelandorientierung und die Vielfalt der Programme könnten indessen die Ressourcen und die Organisationsgröße sein.

Um diese Überlegungen zu prüfen, bilden wir einen »Integrationsindex «. Dieser summative Index beinhaltet die folgenden zusammengefassten Variablen: Teilnahme von Deutschen an den Programmen (wenigstens vereinzelte Teilnahme, ja/nein), Anteil von Frauen an nichtreligiösen Angeboten für Erwachsene (bis $30 \%$ /über 30 \%), Existenz von Zielgruppenangeboten für Erwachsene (ja/nein), religiöse Veranstaltungen auf Deutsch oder mit deutscher Übersetzung (ja/nein), Durchführung von Integrationsprojekten, die von deutscher Seite finanziert wurden (ja/nein). Zu diesen dichotom codierten Bestandteilen wurde zusätzlich eine metrische Variable hinzugerechnet, die aus der Anzahl unterschiedlicher nichtreligiöser Angebote für Jugendliche und Erwachsene gebildet wurde. Um möglichst hohe Fallzahlen zu gewährleisten, wurde die Aufnahme gefilterter Fragen in den Index vermieden. Der so gebildete metrische Index umfasst einen Wertebereich von 0 bis 1 , wobei 0 eine geringe Integrationsorientierung und 1 eine hohe Integrationsorientierung bedeutet. Folgend stellen wir den Mittelwert des Integrationsindex für unterschiedliche Gruppen von Gemeinden dar: 61

61 Zur Berechnung der Zusammenhänge wurden auch für die erklärenden Variablen weitere Indexbildungen vorgenommen: ein summativer metrischer Index der Anzahl unterschiedlicher religiöser Angebote, der in 4 Kategorien umgewandelt wurde (nicht für Aleviten) mit den Ausprägungen wenig, eher wenig, eher hohe und hohe Vielfalt der Angebote; ein metrischer summativer Index der Ressourcen, bestehend aus Vorhandensein eines eigenen Imams bzw. Dedes (ja/nein), Existenz eigener Räumlichkeiten (ja/nein), Existenz von Räumlichkeiten für Freizeit- und Bildungsangebote (ja/nein), Immobilienbesitz (ja/nein), geplante Bauprojekte (ja/nein) und Anzahl von Beauftragten/Abteilungen, der in vier Kategorien zusammengefasst wurde $(0=$ keine Ressourcen, $3=$ hohe Ressourcenausstattung); je höher die Ausprägung dieser Indizes, desto zahlreicher die Angebote bzw. desto größer die Ressourcen. Die Verbandszugehörigkeit umfasst auch Mehrfachnennungen; die Gemeinden, die mehrere Verbandszugehörigkeiten angaben, sind dann in mehreren Kategorien enthalten (zur Mehrfachnennung bei der Verbandszugehörigkeit siehe oben).

Leviathan, 40. Jg., 1/2012 
Tabelle 4: Mittelwert Integrationsindex nach Merkmalen der Gemeinden

\begin{tabular}{|c|c|}
\hline & Mittelwert \\
\hline Glaubensrichtung $(\mathrm{N}=1.102)$ & 0,4974 \\
\hline Sunnitisch & 0,4901 \\
\hline Alevitisch & 0,5536 \\
\hline Herkunft $* * 62(N=1.102)$ & 0,4974 \\
\hline Türkei & 0,4993 \\
\hline Bosnien & 0,5146 \\
\hline Keine dominierende Gruppe & 0,5328 \\
\hline Verbandsmitgliedschaft $(\mathrm{N}=1.102)$ & 0,4974 \\
\hline DITIB & 0,4866 \\
\hline IGMG & 0,5044 \\
\hline VIKZ & 0,4874 \\
\hline $\mathrm{AABF}$ & 0,5422 \\
\hline Keine Verbandsmitgliedschaft & 0,5050 \\
\hline Insgesamt erreichte Klientel $(N=1.102)$ & 0,4974 \\
\hline Bis 20 & 0,4382 \\
\hline 21 bis 50 & 0,4532 \\
\hline 51 bis 200 & 0,5009 \\
\hline 201 bis 500 & 0,5295 \\
\hline Mehr als 500 & 0,6019 \\
\hline
\end{tabular}

62 Wir beschränken uns hier auf die Auswertung der bosnischen, türkischen und nicht durch eine bestimmte Herkunft dominierten Gemeinden. Einerseits sind Bosnien und die Türkei die wichtigsten Herkunftsländer, andererseits ist der Islam in beiden Ländern so unterschiedlich ausgeprägt, dass potenziell Effekte auf den Index zu erwarten wären. 


\begin{tabular}{|l|r|}
\hline & \multicolumn{1}{|c|}{ Mittelwert } \\
\hline Ausprägung Ressourcenindex $(\mathrm{N}=1.069)$ & 0,4987 \\
\hline Keine Ressourcen & 0,3256 \\
\hline Mittlere Ressourcenausstattung & 0,4304 \\
\hline Hohe Ressourcenausstattung & 0,5346 \\
\hline & 0,4946 \\
\hline Vielfalt religiöser Angebote $(\mathrm{N}=1.049)$ & 0,3797 \\
\hline Wenig Vielfalt & 0,4246 \\
\hline Eher wenig Vielfalt & 0,4997 \\
\hline Eher hohe Vielfalt & 0,5135 \\
\hline Hohe Vielfalt & \\
\hline
\end{tabular}

* Inkl. der Aleviten, für die eine türkische Herkunft angenommen wurde.

Der Integrationsindex zeigt, dass die Gemeinden mit Ausprägungen zwischen 0,3256 und 0,6019 deutlich differenziert sind. Bei der Betrachtung der Mittelwerte des Integrationsindex nach den unterschiedlichen Erklärungsvariablen fällt auf, dass - wie vermutet - sich dieser am deutlichsten nach Ressourcen und Größe der Gemeinden unterscheidet, wobei sich der Index mit zunehmender Größe und steigenden Ressourcen positiv entwickelt. Entgegen der These von einer Konkurrenz zwischen religiösen Angeboten und integrationsrelevanten Aktivitäten steigt mit der Vielfalt der religiösen Angebote tendenziell auch der Integrationsindex - vermutlich weil beides von der Ressourcenausstattung beeinflusst wird. Demgegenüber wirken sich Verbandszugehörigkeit, Herkunft und Glaubensrichtung eher gering aus, wobei aber sowohl mit Blick auf die Glaubensrichtung (0,5536 statt 0,4974 im Durchschnitt aller Gemeinden) wie auf die Verbandszugehörigkeit die alevitischen Organisationen etwas bessere Werte erzielen als die anderen. Die Annahme der größeren Offenheit und Integrationsorientierung der alevitischen Gruppe bestätigt sich teilweise auch in der Angebotsstruktur ihrer Organisationen, wobei die Abweichung vom Durchschnitt aber nicht sehr ausgeprägt ist. Bemerkenswert ist zudem der sehr geringe Unterschied der Indexwerte der drei großen sunnitischen Moscheeverbände.

Dass es keine Konkurrenz zwischen Integrationsorientierung und religiösen Angeboten gibt, wird noch einmal deutlich, wenn man den Mittelwert nur für die Moscheegemeinden mit Korankursen bzw. mit Islamunterricht betrachtet: Diese erzielen einen genau durchschnittlichen Wert beim Integrationsindex (0,4977 gegenüber 0,4946 bei allen 1.049 ausgewerteten Moscheen). Zugleich steigert das Vorhandensein von Korankursen bzw. Islamunterricht die Wahrscheinlichkeit, dass eine Moschee auch deutsche Sprachkurse anbietet (Cramers V: 0,118; Signifikanz: < $0,001)$. 
Wir hatten vermutet, dass letztendlich die Ressourcenausstattung und die Größe der Gemeinden die entscheidende Erklärung für die Ausprägung des Integrationsindex liefern. Um dies zu prüfen, wurde eine multivariate logistische Regressionsanalyse durchgeführt. ${ }^{63}$

63 Mit dieser statistischen Methode kann bestimmt werden, ob der Unterschied auf der Glaubensrichtung und der dominierenden Herkunft der Mitglieder basiert, auf der Mitgliedschaft in bestimmten Verbänden oder auf die Größe der Gemeinde und ihre Ressourcen zurückzuführen ist. Mit der logistischen Regression werden Erklärungsmodelle generiert und schrittweise um verschiedene mögliche Einflussvariablen ergänzt, um Ursachen für Unterschiede ausfindig zu machen. Aus dem Integrationsindex wurde eine dichotome Variable (geringer Grad der Offenheit $=0$, hoher Grad der Offenheit $=1$ ) als abhängige Variable gebildet. Als erklärende, unabhängige Variablen wurden im ersten Schritt (Modell 1) die Glaubensrichtung und die dominierende Herkunft in das Modell aufgenommen. Im zweiten Schritt (Modell 2) wurden die erklärenden Variablen um die Zugehörigkeit zu verschiedenen Verbänden ergänzt, im dritten Schritt (Modell 3) um die Gemeindegröße und die verfügbaren Ressourcen. Die logistische Regression berechnet die Wahrscheinlichkeit, mit der die jeweiligen Variablenausprägungen im Vergleich zu einer zu definierenden Referenzgruppe einen hohen Integrationsindex aufweisen. Diese Wahrscheinlichkeit wird durch den Regressionskoeffizienten RK (Exp.(b)) angegeben. Bei einem Wert höher als 1 ist die Wahrscheinlichkeit, einen hohen Wert im Integrationsindex aufzuweisen, größer als in der Referenzgruppe, bei einem Wert unter 1 ist diese Wahrscheinlichkeit geringer als in der Referenzgruppe. Der Zusammenhang zwischen der abhängigen und der unabhängigen Variablen hat nur dann statistisch eine Erklärungskraft, wenn das Signifikanzniveau (oder die Irrtumswahrscheinlichkeit) kleiner als 0,05 (bzw. 5 \%) ist. Bei einem höheren Signifikanzwert beruht der Zusammenhang möglicherweise auf einem Zufall. Der Effekt zeigt die Richtung des Zusammenhangs an: Ist er positiv, steigt die Wahrscheinlichkeit, ist er negativ, sinkt sie, ist er 0 , besteht kein signifikanter Zusammenhang, d.h. die Ausprägung hat keinen Einfluss. Die Erklärungskraft des jeweiligen Modells wird ebenfalls berechnet (Pseudo R2). Der Wert gibt an, wie viel Varianz der abhängigen Variablen durch das Modell erklärt werden kann. Pseudo $\mathrm{R}^{2}$ kann kein Maximum von 1 erreichen; Modelle, die Werte zwischen 0,2 und 0,4 erzielen, gelten als gute Erklärungsmodelle; vgl. Fromm 2010. Nicht berücksichtigt wurde hier die Anzahl unterschiedlicher religiöser Angebote, da dies bedeutet hätte, die Aleviten in der Regression nicht zu berücksichtigen (da ihnen diese Frage nicht gestellt worden war). Eine alternative Regressionsanalyse ohne Berücksichtigung der Aleviten, aber unter Hinzuziehung der Vielfalt des religiösen Angebots ergab, dass dieses Angebot keinen signifikanten Erklärungsbeitrag für die Ausprägung des Integrationsindex liefert. 
Tabelle 5: Logistische Regression zum Grad der Offenheit/Integrationsorientierung $(\mathrm{N}=765)$

\begin{tabular}{|c|c|c|c|}
\hline \multirow[t]{2}{*}{$\begin{array}{l}\text { Abhängige Variable } \\
\text { Ausprägung Integrationsindex } \\
(0=\text { geringer Grad, } 1=\text { hoher Grad })\end{array}$} & \multirow[b]{2}{*}{$\mathrm{RK}^{*}$} & \multirow[b]{2}{*}{ Effekt $* *$} & \multirow[b]{2}{*}{ Sign. } \\
\hline & & & \\
\hline \multicolumn{4}{|l|}{ Glaubensrichtung } \\
\hline Sunnitisch & \multicolumn{2}{|c|}{ Referenzgruppe } & - \\
\hline Schiitisch & & 0 & - \\
\hline Ahmadi & & 0 & - \\
\hline Gemischt & & 0 & - \\
\hline Alevitisch & & 0 & - \\
\hline \multicolumn{4}{|l|}{ Dominierende Herkunft } \\
\hline Türken & \multicolumn{2}{|c|}{ Referenzgruppe } & - \\
\hline Bosnier & & 0 & - \\
\hline Andere & & 0 & - \\
\hline Keine dominierende Herkunftsgruppe & & 0 & - \\
\hline \multicolumn{4}{|l|}{ Mitgliedschaft DITIB } \\
\hline Keine Mitgliedschaft DITIB & \multicolumn{2}{|c|}{ Referenzgruppe } & - \\
\hline Mitgliedschaft DITIB & & 0 & - \\
\hline \multicolumn{4}{|l|}{ Mitgliedschaft IGMG } \\
\hline Keine Mitgliedschaft IGMG & \multicolumn{2}{|c|}{ Referenzgruppe } & - \\
\hline Mitgliedschaft IGMG & & 0 & - \\
\hline \multicolumn{4}{|l|}{ Mitgliedschaft VIKZ } \\
\hline Keine Mitgliedschaft VIKZ & \multicolumn{2}{|c|}{ Referenzgruppe } & - \\
\hline Mitgliedschaft VIKZ & & 0 & - \\
\hline \multicolumn{4}{|l|}{ Mitgliedschaft AABF } \\
\hline Keine Mitgliedschaft AABF & \multicolumn{2}{|c|}{ Referenzgruppe } & - \\
\hline Mitgliedschaft AABF & & 0 & - \\
\hline \multicolumn{4}{|l|}{ Mitgliedschaft andere Verbände } \\
\hline Keine Mitgliedschaft andere Verbände & \multicolumn{2}{|c|}{ Referenzgruppe } & $*$ \\
\hline Mitgliedschaft andere Verbände & 1,318 & + & $*$ \\
\hline
\end{tabular}




\begin{tabular}{|c|c|c|c|}
\hline \multirow[t]{2}{*}{$\begin{array}{l}\text { Abhängige Variable } \\
\text { Ausprägung Integrationsindex } \\
(0=\text { geringer Grad, } 1=\text { hoher Grad })\end{array}$} & \multirow[b]{2}{*}{$\mathrm{RK}^{*}$} & \multirow[b]{2}{*}{ Effekt $* *$} & \multirow[b]{2}{*}{ Sign. } \\
\hline & & & \\
\hline \multicolumn{4}{|l|}{ Unabhängig } \\
\hline Mitgliedschaft in Verband & \multicolumn{2}{|c|}{ Referenzgruppe } & - \\
\hline Unabhängig & & 0 & - \\
\hline \multicolumn{4}{|l|}{ Ressourcen } \\
\hline Wenige Ressourcen & \multicolumn{2}{|c|}{ Referenzgruppe } & $* * *$ \\
\hline Mittlere Ressourcen & & 0 & - \\
\hline Viele Ressourcen & 2,823 & + & $* * *$ \\
\hline \multicolumn{4}{|l|}{ Gemeindegröße } \\
\hline Bis 20 Personen & \multicolumn{2}{|c|}{ Referenzgruppe } & $* * *$ \\
\hline 21-50 Personen & & 0 & - \\
\hline 51-200 Personen & & 0 & - \\
\hline 201-500 Personen & & 0 & - \\
\hline Mehr als 500 Personen & 3,498 & + & $* *$ \\
\hline Pseudo R² (Nagelkerke) & \multicolumn{3}{|l|}{0,139} \\
\hline
\end{tabular}

$* R K=$ Regressionskoeffizient $($ Exp.(b)).

** Richtung der Wahrscheinlichkeitsänderung: + = im Vergleich zur Referenzgruppe Zunahme der Wahrscheinlichkeit eines hohen Grades an Offenheit/Integrationsorientierung; - = im Vergleich zur Referenzgruppe Abnahme der Wahrscheinlichkeit eines hohen Grades an Offenheit/Integrationsorientierung; $0=$ kein Effekt, da nicht signifikant.

Signifikanzniveaus: $* *<0,001, * * 0,01, *<0,05,-=$ nicht signifikant.

Das Ergebnis zeigt, dass weder die Glaubensrichtung noch die dominierende Herkunft signifikante Zusammenhänge zur Wahrscheinlichkeit aufweisen, einen hohen Wert im Integrationsindex zu erreichen, wenn man wie im dargestellten Modell 3 auch die Ressourcenausstattung und die Gemeindegröße einbezieht. Insbesondere nivelliert sich der Einfluss der alevitischen Religionszugehörigkeit. Auch die Mitgliedschaft in Verbänden hat keinen Einfluss - mit Ausnahme der Mitgliedschaften in anderen als den großen Verbänden. Die Ausstattung mit Ressourcen und die Gemeindegröße wirken sich am deutlichsten aus, wobei schon eine hohe Ausstattung mit Ressourcen notwendig ist, um die Wahrscheinlichkeit zu steigern, einen hohen Wert im Integrationsindex zu erreichen. Ebenso macht sich die Gemeindegröße erst ab 500 Personen signifikant bemerkbar. Insgesamt können mit diesem Modell jedoch nur $14 \%$ der Varianz erklärt werden. Eine alternative Berechnung der Regression unter Ausschluss der Gemeinden mit mehrfacher Verbandsmitgliedschaft 
erbringt insofern ein anderes Ergebnis, als eine Mitgliedschaft im VIKZ den Integrationsindex negativ beeinflusst, während die übrigen Zusammenhänge erhalten bleiben - die deutlich stärker ausgeprägt sind als der Effekt der VIKZ-Mitgliedschaft.

Somit zeigt sich, dass es wenig Anhaltspunkte gibt, dass eine bestimmte Glaubensrichtung, Herkunft oder Verbandszugehörigkeit die Übersetzung von Ressourcen in integrationsrelevante Angebote behindern.

\subsection{Rolle der Verbände 64}

Wir hatten eingangs darauf hingewiesen, dass die Rolle der muslimischen Verbände politisch umstritten ist. Es steht in Frage, inwieweit sie die muslimische Gemeinschaft in Deutschland repräsentieren und als legitime Vertretung anzuerkennen sind. Die uns zur Verfügung stehenden Daten können zu dieser Frage nur sehr bedingt eine Antwort liefern. In der bereinigten Ausgangsdatenbank sind von 2.342 Gemeinden rund 1.200 in den großen bzw. im Koordinierungsrat der Muslime vertretenen Verbänden DITIB, VIKZ, IGMG, Islamrat oder ZMD. Dies entspricht dem Bild von der Rolle der Verbände für die Gemeinden, das Krech für NRW und Spielhaus für Berlin zeichnen. ${ }^{65}$ Auch die bereinigte Datenbank für ganz Deutschland zeigt keine gravierenden Verschiebungen der Situation. Betrachtet man die Zusammenhänge zwischen Verbandszugehörigkeit und Organisationsalter, so ergibt sich dennoch ein vorsichtiger Hinweis darauf, dass von einer langfristigen Schwächung der großen Verbände auszugehen sein könnte, da Neugründungen von Gemeinden vermehrt abseits der etablierten Organisationslandschaft stattfinden. So wurden $22 \%$ derjenigen Gemeinden, die keine Verbandsmitgliedschaft angaben, nach dem Jahr 2000 gegründet, bei den verbandlich organisierten beträgt dieser Anteil nur $11 \%$. Hier besteht ein signifikanter, aber schwacher Zusammenhang zwischen Organisationsalter und fehlender Verbandsmitgliedschaft (Cramers V: 0,166; Signifikanz: < 0,001). Analog bestehen schwache signifikante Korrelationen der Mitgliedschaft bei DITIB, IGMG und VIKZ mit einem höheren Organisationsalter. Die Mitgliedschaft in anderen Verbänden hat keinen signifikanten Effekt. ${ }^{66}$ Ausnahmen sind die alevitischen Gemeinden, die zu 78 \% ab 1990 gegründet wurden und damit deutlich jünger sind als alle übrigen Kategorien. Die Entstehung alevitischer Organisationen in den 1990er Jahren ist dabei den spezifischen Identitätsbildungsprozessen der alevitischen Gemeinschaft geschuldet. ${ }^{67}$

64 Alle Berechnungen in diesem Kapitel beruhen auf tatsächlich erhaltenen Auskünften. Fälle, in denen keine Angaben erfolgten, wurden nicht berücksichtigt.

65 Das ist allerdings nicht unerwartet, weil die Ausgangsdatenbank unter anderem die Verzeichnisse von Krech und Spielhaus beinhaltete.

66 In der Berechnung mit der alternativen Verbandsvariablen (ohne Gemeinden mit Doppelmitgliedschaften) besteht kein signifikanter Zusammenhang zwischen Organisationsalter und IGMG-Mitgliedschaft mehr, dafür zeigen sich aber schwache signifikante $\mathrm{Zu}$ sammenhänge zwischen Organisationsalter und der Mitgliedschaft in anderen Verbänden; sie sind überdurchschnittlich jung.

67 Siehe zu dieser Entwicklung ausführlich Sökefeld 2008 c, S. 272.

Leviathan, 40. Jg., 1/2012 
Unsere Daten sind insbesondere geeignet, die Bedeutung der Verbandsmitgliedschaft für die Ressourcen und die Angebote der Gemeinden zu analysieren. Oben wurde dargestellt, dass größere Gemeinden eine eher bessere Ressourcenausstattung vorweisen können, was wiederum das Vorliegen integrationsrelevanter Merkmale begünstigt. Ein - allerdings eher schwacher - signifikanter Zusammenhang besteht auch zwischen Gemeindegröße und Verbandszugehörigkeit. Unter den DITIB-, IGMG- und VIKZ-Mitgliedern sind überproportional häufig größere Gemeinden vorzufinden - gemessen an der Klientel, die sie mit ihren Angeboten insgesamt erreichen -, bei den Angehörigen kleinerer Verbände dagegen finden sich überproportional häufig kleinere Gemeinden. Bei den alevitischen und den verbandsunabhängigen Gemeinden zeigt sich kein solcher signifikanter Zusammenhang. ${ }^{68}$ Auf den Ressourcenindex wirkt die Verbandszugehörigkeit schwach und signifikant nur im Falle der DITIB und der verbandsunabhängigen Gemeinden. Letztere sind eher ressourcenschwach (Cramers V: 0,190; Signifikanz: < 0,001), DITIB-Mitglieder eher ressourcenstark (Cramers V: 0,168; Signifikanz: <0,001).

Verbandsunabhängige Gemeinden machen tendenziell weniger nichtreligiöse Angebote als die Gemeinden der anderen Kategorien, wobei auch dieser Zusammenhang nur schwach ist (Cramers V: 0,130; Signifikanz: < 0,001). Bei der Anzahl unterschiedlicher religiöser Angebote der Gemeinden sind die Zusammenhänge etwas anders. Die Zugehörigkeit zu einem der drei großen Verbände begünstigt solche Angebote schwach und signifikant, während der Effekt bei der Zugehörigkeit zu einem der kleineren Verbände noch etwas schwächer ist. Der ausgeprägteste $\mathrm{Zu}$ sammenhang besteht aber zwischen Nichtmitgliedschaft in einem Verband und dem Mangel an religiösen Angeboten (Cramers V: 0,341; Signifikanz: <0,001). ${ }^{69}$

Es zeigt sich, dass den großen Verbänden momentan eine wichtige Rolle für die »religiöse Grundversorgung « zukommt. Von verbandsfreien Gemeinden kann eine solche Grundversorgung nur in begrenztem Umfang geleistet werden, was an den geringeren zur Verfügung stehenden Ressourcen und eventuell weniger starker Nachfrage aufgrund tendenziell kleinerer Nutzerzahlen liegen mag. Ressourcen sind zugleich eine Voraussetzung für religiöse wie nichtreligiöse Aktivitäten. Allerdings übersetzt sich das Vorhandensein von Ressourcen nicht ohne weiteres in die Ausweitung von Angeboten. Das gilt freilich für viele andere Kontexte in gleicher Weise und ist ein Grundproblem speziell förderpolitischer Interventionen.

Die (schwachen) Hinweise auf ein mögliches langfristiges Erstarken des verbandsunabhängigen Sektors, die sich aus der vergleichsweise großen Zahl entsprechender Neugründungen ergeben, gilt es im nächsten Kapitel noch weiter zu verfolgen. Speziell ist die Frage von Interesse, ob diese Entwicklung tatsächlich daraus resultiert, dass die etablierten Organisationen sozialem und intergenerationalem Wandel eventuell immer weniger gerecht werden.

68 In der Berechnung mit der alternativen Verbandsvariablen (ohne Gemeinden mit Doppelmitgliedschaften) verschwinden diese signifikanten Zusammenhänge. Hier ergibt sich hingegen nur für die unabhängigen Gemeinden ein signifikanter Zusammenhang.

69 Berechnung ohne alevitische Gemeinden, für die kein Index religiöser Angebote gebildet wurde. 


\subsection{Die Gemeinden im intergenerationalen Wandel}

Aufschluss über sozialen und intergenerationalen Wandel in den Gemeinden geben die Generationszugehörigkeit der Vereinsführung und der Besucher, das Vorhandensein von Programmen für Jugendliche sowie die Sprache, in der das religiöse Programm angeboten wird.

In rund der Hälfte aller befragten Gemeinden hat sich bereits ein intergenerationaler Wandel insofern vollzogen, als der Vereinsvorsitzende der zweiten Generation der Einwanderer entstammt (59\%) bzw. die Nachfolgegeneration unter den Besuchern dominiert (46\%). In exakt einem Drittel der Gemeinden dominiert keine bestimmte Einwanderergeneration. Die Dominanz einer bestimmten Einwanderergeneration wird das Gemeindeleben nicht vollkommen prägen. Generell sind in den meisten Organisationen grundsätzlich so gut wie alle Einwanderergruppen präsent, mit Ausnahme von Flüchtlingen und Besuchern ohne Migrationshintergrund, also deutschstämmigen Muslimen, die in weniger als der Hälfte der Gemeinden vorkommen - aber selbst letztere finden sich in $43 \%$ der Organisationen. Die Gemeinden sind nicht klar als Erstgenerations- oder Nachfolgegenerationsorganisationen profiliert. Für diese Interpretation spricht auch, dass ein Zusammenhang zwischen der Generationszugehörigkeit des Vorsitzenden und der Generationszugehörigkeit der jeweils dominierenden Gruppe der Besucher zwar vorhanden, aber schwach ist. Tendenziell haben von der Nachfolgegeneration besuchte Moscheen auch entsprechende Vorsitzende, ohne dass dies jedoch die Regel wäre (Cramers V: 0,180; Signifikanz: <0,01). ${ }^{70}$ Ebenso sind die Zusammenhänge von Organisationsalter und Einwanderungshintergrund der jeweils dominierenden Gruppe von Besuchern (Cramers V: 0,099; Signifikanz < 0,05) bzw. des Vorsitzenden (Cramers V: 0,1; Signifikanz $<0,01)$ nur schwach. Intergenerationaler Wandel findet damit durchaus innerhalb der etablierten Gemeinden statt und nicht zwangsläufig dadurch, dass etablierte Gemeinden durch »jüngere « Gründungen ersetzt werden. Für die vermehrte Gründung verbandsfreier Gemeinden ab dem Jahr 2000 sind damit eher andere soziale Prozesse verantwortlich als der intergenerationale Wandel in der muslimischen Bevölkerung.

Als weitere wichtige Merkmale des Wandels in den befragten Gemeinden werden die Existenz spezieller Angebote für Jugendliche sowie das Vorhandensein deutschsprachiger religiöser Angebote betrachtet, da davon auszugehen ist, dass sich über die Zeit die Kenntnisse der Herkunftssprache verlieren. Analog zum oben dargestellten »Integrationsindex « bilden wir aus diesen Variablen einen »Erneuerungsindex «. Dieser summative metrische Index beinhaltet die folgenden Variablen: religiöse Veranstaltungen auf Deutsch oder mit deutscher Übersetzung (ja/nein), Vorsitzender Angehöriger der Nachfolgegeneration oder ohne Migrationshintergrund (ja/nein), dominierende Gruppe unter den Mitgliedern und Besuchern aus der Nachfolgegeneration oder ohne Migrationshintergrund (ja/nein). $\mathrm{Zu}$ diesen dichotomen

70 Einschränkend muss darauf hingewiesen werden, dass dieser Zusammenhang aus technischen Gründen eher schwach ausfallen muss, da kleinere Gruppen durchaus hier und da Vorstände, allerdings kaum dominierende Herkunftsgruppen stellen können.

Leviathan, 40. Jg., 1/2012 
Bestandteilen kommt eine zusätzliche metrische Variable, die aus der Anzahl unterschiedlicher nichtreligiöser Angebote für Jugendliche gebildet wurde. Der so entstandene metrische Index umfasst einen Wertebereich von 0 bis 1 , wobei 0 eine geringe Erneuerungsorientierung und 1 eine hohe Erneuerungsorientierung bedeutet.

Die Ausprägung dieses Index betrachten wir entsprechend der eingangs begründeten Annahmen zunächst nach Verbandszugehörigkeit $(\mathrm{N}=1.076)$ und Herkunftsdominanz bzw. Herkunftshomogenität $(\mathrm{N}=879)$ der Gemeinden.

Es zeigt sich, dass der Erneuerungsindex in beiden Betrachtungen zwischen den unterschiedlichen Kategorien nur gering variiert, und zwar in eine eher unerwartete Richtung. Der Indexdurchschnitt aller ausgewerteten Fälle bei der Herkunftsbetrachtung beträgt 0,5440 und bei der Verbandszugehörigkeit 0,5303 . Speziell bei denjenigen Gemeinden, in denen keine Herkunftsgruppe oder seltenere Herkunftsgruppen (also nicht Türken oder Bosnier) dominieren, und bei den Gemeinden ohne Verbandsmitgliedschaft ist der Erneuerungsindex unterdurchschnittlich ausgeprägt $(0,5079,0,4907$ bzw. 0,5065), am höchsten ist er bei der IGMG $(0,5965)$ und beim VIKZ (0,5952), wobei die Ergebnisse in Bezug auf die IGMG durchaus mit dem Kenntnisstand über diese Organisation übereinstimmen - die IGMG hat sich, so die Beobachtung, aufgrund interner und äußerer Einflüsse in der jüngeren Zeit stark verändert und war als Organisation in der Lage, den Wandel ihrer Klientel mitzuvollziehen. ${ }^{71}$ Allerdings ergibt eine alternative Berechnung des Erneuerungsindex unter Ausschluss von Gemeinden mit Doppelmitgliedschaften einen nur durchschnittlichen Mittelwert für die IGMG, während die Werte der anderen Kategorien nur gering variieren. Der Befund der besonderen Erneuerung der IGMG bleibt also unsicher - im Gegensatz zum Befund über die zum VIKZ zählenden Gemeinden.

Die Auswertung stützt zunächst wiederum die Interpretation, dass sich ein Wandel zur Erneuerung des muslimischen Lebens durchaus im Rahmen etablierter Verbandsstrukturen abspielt. Auch das Gemeindealter korreliert nicht mit der Ausprägung des Erneuerungsindex. Allerdings liegt die Vermutung nahe, dass - wie schon beim Integrationsindex - die Ressourcenausstattung eine wichtige moderierende Variable ist, die als Voraussetzung für die Erneuerung der Gemeinden zu begreifen wäre. So besteht dann auch ein schwacher Zusammenhang zwischen der Ressourcenausstattung der Gemeinden und ihrem Erneuerungsgrad (Gamma: 0,108; Signifikanz: <0,001), wobei, wie oben gezeigt, verbandsunabhängige Gemeinden tendenziell ressourcenschwächer sind. Hierin mag eine Erklärung für die vergleichsweise schwache Ausprägung des Erneuerungsindex bei den verbandsunabhängigen Gemeinden liegen. Es ist zu vermuten, dass insbesondere Angebote für Jugendliche als Bestandteile des Index ressourcenabhängig sind, die Generationenzugehörigkeit

71 Allerdings ist zu beachten, dass auch viele der durch eine Gruppe dominierten Gemeinden im Ergebnis herkunftsheterogen sind. Ein signifikanter Zusammenhang zwischen der tatsächlichen Nennung nur einer Herkunftsgruppe und einem niedrigen Erneuerungsindex besteht sehr wohl, aber schwach (Gamma: 0,148; Signifikanz: <0,001), was eher den Erwartungen eines Zusammenhangs von Herkunftsheterogenität und Erneuerung entspricht. 
des Vorsitzenden und die dominierende Gruppe sowie die Verwendung der deutschen Sprache hingegen nicht. Eine Korrelationsanalyse bestätigt diese Vermutung weitgehend. Ein höherer Ressourcenindex geht einher mit vielfältigeren Angeboten für Jugendliche (Gamma: 0,297; Signifikanz: <0,001), kein Zusammenhang besteht zu religiösen Programmen in deutscher Sprache. Zusammenhänge zwischen Ressourcenindex und der Generationenzugehörigkeit der dominierenden Gruppe (Cramers V: 0,092; Signifikanz: < 0,01) sowie des Vorsitzenden (Cramers V: 0,109; Signifikanz: < 0,05) sind zwar vorhanden, aber sehr schwach, da Gemeinden, in denen Angehörige aus der zweiten Generation überwiegen, tendenziell über mehr Ressourcen verfügen.

Für die bei der DITIB organisierten Gemeinden einerseits und für die verbandsunabhängigen Gemeinden andererseits konnten wir oben signifikante Unterschiede bei der Ressourcenausstattung zeigen. Vergleichen wir nun den Erneuerungsindex für DITIB- und die verbandsfreien Gemeinden unter Berücksichtigung der Ausprägung des Ressourcenindex, so wird deutlich, das sich bei den verbandsunabhängigen Gemeinden ein Zusammenhang zwischen dem Vorhandensein von Ressourcen und Erneuerung zeigt (Gamma: 0,108; Signifikanz: < 0,001), nicht jedoch bei DITIBGemeinden.

Tabelle 6: Erneuerungsindex nach Ressourcenindex ${ }^{72}$ bei DITIB- und verbandsunabhängigen Gemeinden

\begin{tabular}{|l|r|r|r|r|}
\hline & \multicolumn{2}{|c|}{ DITIB } & \multicolumn{2}{c|}{ unabhängig } \\
\hline Ressourcen & $\begin{array}{c}\text { Mittelwert Er- } \\
\text { neuerungsindex }\end{array}$ & $N$ & $\begin{array}{c}\text { Mittelwert Er- } \\
\text { neuerungsindex }\end{array}$ & N \\
\hline Keine & 0,375 & 1 & 0 & 40 \\
\hline Gering & 0,5724 & 39 & 0,4881 & 76 \\
\hline Mittel & 0,5401 & 88 & 0,4813 & 119 \\
\hline Hoch & 0,5372 & 357 & 0,5525 & 237 \\
\hline Total & 0,5402 & 485 & 0,5141 & \\
\hline
\end{tabular}

Speziell das Vorhandensein sehr vieler Ressourcen wirkt bei den unabhängigen Gemeinden günstig auf den Erneuerungsindex, der in diesem Fall überdurchschnittlich ausgeprägt ist, auch ausgeprägter als bei den mit DITIB verbundenen Gemeinden. Dass sich die Erneuerung der DITIB-Gemeinden neutral gegenüber der Ressourcenausstattung erweist, dürfte darin begründet sein, dass die DITIB im Vergleich zu den unabhängigen Gemeinden durch den - eher ressourcenunabhängigen

72 Ressourcenindex in 4 Stufen: $0-0,24,0,25-0,49,0,5-0,74,0,75-1,0$.

Leviathan, 40. Jg., 1/2012 
- Generationenwechsel Indexpunkte in höherer Größenordnung erzielt. ${ }^{73}$ Eine bessere Ressourcenausstattung scheint damit eher zur Erneuerung bei den verbandsfreien Gemeinden (über die Ausweitung der Jugendarbeit) zu führen als bei Gemeinden, die bei der DITIB organisiert sind. Nur im ersteren Fall würde eine bessere Ressourcenausstattung positiv auf den Erneuerungsindex wirken.

Insgesamt ist die Frage nach der Rolle der etablierten Verbandsstrukturen einerseits und verbandsfreier Organisationen andererseits für die Erneuerung der Organisationen in Deutschland sehr differenziert zu beantworten. Intergenerationeller Wandel findet auch in den etablierten Organisationen in großem Umfang statt, so dass die vermehrte Neugründung unabhängiger Gemeinden nicht einfach als Reaktion auf eine mangelnde Wandlungsfähigkeit der großen Verbände erklärt werden kann. Allerdings wird deutlich, dass das Erneuerungspotenzial der verbandsunabhängigen Gemeinden als groß einzuschätzen ist. Die deutsche Sprache ist hier schon heute überdurchschnittlich weit verbreitet, was in der geringeren Dominanz einzelner Herkunftsgruppen begründet ist.

Abschließend ist noch auf die Frage nach den grenzüberschreitenden Kontakten der Gemeinden und dem Grad ihrer Erneuerung einzugehen. Anhand der Daten war es möglich zu untersuchen, ob ein Zusammenhang zwischen dem tendenziellen Bedeutungsverlust des Herkunftslands im intergenerationalen Wandel und den Herkunftslandkontakten von Funktionären der befragten Organisationen besteht, sodass Herkunftslandkontakte eventuell durch paneuropäische, auf migrantische oder muslimische Identitäten rekurrierende Netzwerke ersetzt werden.

Die Ansprechpartner in den Gemeinden wurden daher gefragt, welche Kontakte die Organisationen zu anderen Organisationen desselben Glaubens unterhalten. Hier zeigt sich zunächst, dass Landesgrenzen überschreitende Kontakte der Gemeinden eher schwach ausgeprägt sind, viel schwächer, als man es mit Blick auf Migrantenorganisationen vielleicht erwarten würde. Nur eine Minderheit der Organisationen unterhält überhaupt Landesgrenzen überschreitende Kontakte, wobei sich Diaspora- und Herkunftslandkontakte ungefähr die Waage halten $(15 \%$ bzw. $19 \%) .{ }^{74}$ Entsprechende Kontakte zu anderen Gemeinden innerhalb Deutschlands unterhalten demgegenüber $73 \%$ aller Gemeinden. Weder Diaspora- noch Herkunftslandkontakte korrelieren dabei mit dem Erneuerungsindex, wohl aber besteht ein Zusammenhang zwischen Ressourcen und Herkunftslandkontakten (Cramers V: 0,257; Signifikanz < 0,01), wobei Ressourcen Herkunftslandkontakte begünstigen. Zudem korrelieren Diaspora-Kontakte ins europäische Ausland (ohne Herkunftsland) und Herkunftslandkontakte der Gemeinden untereinander stark, mit europäischen Diaspora-Kontakten steigt auch die Wahrscheinlichkeit der Her-

73 Hier besteht in gewissem Umfang ein Validitätsproblem des Messinstruments, da die Zugehörigkeit zur Nachfolgegeneration eher für Gruppen mit Gastarbeiterherkunft, zuvorderst für in türkischen Gemeinden Organisierte, zutrifft. Diese können also im Erneuerungsindex besser »punkten « als Herkunftsgruppen mit kürzerer Aufenthaltsdauer, die sich per se noch nicht durch intergenerationalen Wandel erneuert haben konnten.

74 Die geringen europäischen Kontakte entsprechen den oben zitierten Befunden von Klause 2006, S. 145. 
kunftslandkontakte (Cramers V: 0,469; Signifikanz: < 0,001). 50 \% der Gemeinden mit Herkunftslandkontakten unterhalten auch grenzüberschreitende DiasporaKontakte. Dieser Befund spricht sehr deutlich gegen die Annahme, dass im intergenerationalen Wandel Herkunftslandkontakte durch Diaspora-Kontakte abgelöst werden.

Zur Prüfung der signifikanten und eigenständig erklärenden Variablen der europäischen Diaspora-Netzwerkbildung (europäisches Netzwerk vorhanden/nicht vorhanden) wurde wiederum eine multivariate logistische Regressionsanalyse durchgeführt. Als erklärende, unabhängige Variablen wurden - orientiert am eingangs dargestellten Forschungsstand - im ersten Schritt die Glaubensrichtung und das Dominieren einer bestimmten Herkunft der Gemeindemitglieder, die Mitgliedschaft in einzelnen Verbänden und die dominierende Generationszugehörigkeit der Gemeindemitglieder in das Modell aufgenommen (Modell 1). Im zweiten Schritt (Modell 2) wurden die erklärenden Variablen um die Gemeindegröße und die verfügbaren Ressourcen ergänzt.

Tabelle 7: Logistische Regression zum europäischen Netzwerk $(N=759)$

\begin{tabular}{|c|c|c|c|}
\hline \multirow[t]{2}{*}{$\begin{array}{l}\text { Abhängige Variable } \\
\text { Vorhandensein eines europäischen Netzwerks } \\
(0=\text { Nein, } 1 \text { = Ja })\end{array}$} & \multirow[b]{2}{*}{$\mathrm{RK}^{*}$} & \multirow[b]{2}{*}{ Effekt $* *$} & \multirow[b]{2}{*}{ Sign. } \\
\hline & & & \\
\hline \multicolumn{4}{|l|}{ Glaubensrichtung } \\
\hline Sunnitisch & \multicolumn{2}{|c|}{ Referenzgruppe } & $*$ \\
\hline Schiitisch & - & 0 & - \\
\hline Ahmadi & - & 0 & - \\
\hline Sufi & - & 0 & - \\
\hline Gemischt & - & 0 & - \\
\hline Alevitisch & 2,262 & + & \\
\hline \multicolumn{4}{|l|}{ Dominierende Herkunftsgruppe } \\
\hline$J a$ & \multicolumn{2}{|c|}{ Referenzgruppe } & - \\
\hline Nein & & 0 & - \\
\hline \multicolumn{4}{|l|}{ Dominierende Generation } \\
\hline Erste Generation & \multicolumn{2}{|c|}{ Referenzgruppe } & - \\
\hline 2. Generation oder ohne Migrationshintergrund & & 0 & - \\
\hline \multicolumn{4}{|l|}{ Mitgliedschaft DITIB } \\
\hline Nein & \multicolumn{2}{|c|}{ Referenzgruppe } & $* * *$ \\
\hline $\mathrm{Ja}$ & 0,412 & - & $* * *$ \\
\hline
\end{tabular}




\begin{tabular}{|c|c|c|c|}
\hline \multirow[t]{2}{*}{$\begin{array}{l}\text { Abhängige Variable } \\
\text { Vorhandensein eines europäischen Netzwerks } \\
(0=\text { Nein, } 1=\mathrm{Ja})\end{array}$} & \multirow[b]{2}{*}{$\mathrm{RK}^{*}$} & \multirow[b]{2}{*}{ Effekt ${ }^{* *}$} & \multirow[b]{2}{*}{ Sign. } \\
\hline & & & \\
\hline \multicolumn{4}{|l|}{ Mitgliedschaft IGMG } \\
\hline Nein & \multicolumn{2}{|c|}{ Referenzgruppe } & $* *$ \\
\hline $\mathrm{Ja}$ & 1,920 & + & $* *$ \\
\hline \multicolumn{4}{|l|}{ Mitgliedschaft VIKZ } \\
\hline Nein & \multicolumn{2}{|c|}{ Referenzgruppe } & $* *$ \\
\hline $\mathrm{Ja}$ & 0,397 & - & $* *$ \\
\hline \multicolumn{4}{|l|}{ Mitgliedschaft AABF } \\
\hline Nein & \multicolumn{2}{|c|}{ Referenzgruppe } & - \\
\hline $\mathrm{Ja}$ & & 0 & - \\
\hline \multicolumn{4}{|l|}{ Mitgliedschaft andere Verbände } \\
\hline Nein & \multicolumn{2}{|c|}{ Referenzgruppe } & - \\
\hline $\mathrm{Ja}$ & & 0 & - \\
\hline \multicolumn{4}{|l|}{ Unabhängig } \\
\hline Nein & \multicolumn{2}{|c|}{ Referenzgruppe } & - \\
\hline $\mathrm{Ja}$ & & 0 & - \\
\hline \multicolumn{4}{|l|}{ Ressourcen } \\
\hline Wenige Ressourcen & \multicolumn{2}{|c|}{ Referenzgruppe } & $* *$ \\
\hline Mittlere Ressourcen & & 0 & - \\
\hline Viele Ressourcen & 2,695 & + & $*$ \\
\hline \multicolumn{4}{|l|}{ Gemeindegröße } \\
\hline Bis 20 Personen & \multicolumn{2}{|c|}{ Referenzgruppe } & - \\
\hline 21-50 Personen & & 0 & - \\
\hline 51-200 Personen & & 0 & - \\
\hline 201-500 Personen & & 0 & - \\
\hline Mehr als 500 Personen & & 0 & - \\
\hline Pseudo R² (Nagelkerke) & \multicolumn{3}{|l|}{0,131} \\
\hline
\end{tabular}

$* R K=$ Regressionskoeffizient $($ Exp.(b)).

** Richtung der Wahrscheinlichkeitsänderung: + = im Vergleich zur Referenzgruppe Zunahme der Wahrscheinlichkeit eines hohen Grades an Offenheit; - = im Vergleich zur Referenzgruppe Abnahme der Wahrscheinlichkeit eines hohen Grades an Offenheit; $0=$ kein Effekt, da nicht signifikant.

Signifikanzniveaus: $* * 00,001, * * 0,01, *<0,05,-=$ nicht signifikant. 
Es zeigt sich, dass weder die dominierende Herkunft oder die dominierende Generation noch die Gemeindegröße signifikante eigenständige Zusammenhänge zur Wahrscheinlichkeit, über ein europäisches Diaspora-Netzwerk zu verfügen, aufweisen. Allerdings steigert die Zugehörigkeit der Gemeinden zum Alevitentum die Wahrscheinlichkeit paneuropäischer Netzwerke deutlich, analog zum Forschungsstand zu dieser Gruppe und ihren Organisationen. ${ }^{75}$ Signifikant ist auch der $\mathrm{Zu}$ sammenhang zur Mitgliedschaft bei DITIB, IGMG und beim VIKZ: Gehören die Gemeinden der DITIB bzw. dem VIKZ an, haben sie wahrscheinlicher ein europäisches Diaspora-Netzwerk, als wenn sie nicht der DITIB bzw. dem VIKZ angehören. Speziell bei IGMG-Mitgliedschaft ist die Wahrscheinlichkeit eines europäischen Netzwerks deutlich erhöht. Dieser Zusammenhang ist sogar noch erheblich stärker als bei den alevitischen Gemeinden. Die Ausstattung mit Ressourcen wirkt sich stark und signifikant aus, wobei im Vergleich zu einer geringen Ressourcenausstattung eine mittlere Ausstattung nicht ausreicht, sondern schon eine gute Ausstattung mit Ressourcen Voraussetzung zu sein scheint, um ein europäisches Diaspora-Netzwerk zu ermöglichen. Insgesamt können mit Modell 2 jedoch nur $13 \%$ der Varianz des Vorhandenseins eines europäischen Diaspora-Netzwerks erklärt werden.

Die These der Europäisierung der Organisationen in der Folge ihrer Erneuerung findet damit in den uns vorliegenden Daten keine Nahrung. Dies dürfte zuvorderst damit zu tun haben, dass grenzüberschreitende Kontakte der Organisationen überhaupt ein eher seltenes Phänomen sind. Zugleich erweisen sich - nicht unplausiblerweise - solche Kontakte als ressourcenabhängig. Eine Erklärung für die grenzüberschreitenden Kontakte liegt damit nicht im Wandel der Klientel, sondern in der Organisation selbst. Dieser Befund macht noch einmal deutlich, dass Organisationshandeln der gesonderten Betrachtung bedarf. ${ }^{76}$ Sozialintegration von Einwanderern und Aktivitäten »ihrer " Organisationen müssen nicht in einem direkten $\mathrm{Zu}$ sammenhang stehen.

\section{Fazit}

Unsere Studie schafft erstmalig einen umfassenden empirischen Zugang zur muslimischen bzw. alevitischen Organisationslandschaft in Deutschland und belegt deutlich, dass in Wissenschaft und Öffentlichkeit vorherrschende, oft auf Vermutungen beruhende Vorstellungen über die Situation des organisierten Islams und seine zukünftige Entwicklung differenzierungsbedürftig sind. Es lassen sich zwar viele erwartete Zusammenhänge und Entwicklungen nachweisen, nicht jedoch als allgemein gültige Phänomene, sondern nur unter bestimmten Randbedingungen. Als wichtigste dieser Randbedingungen identifiziert die Studie das Vorhandensein personeller, infrastruktureller und finanzieller Ressourcen, die Voraussetzungen dafür

75 Bezogen auf die Mitgliedschaft im AABF ist der Zusammenhang aufgrund der noch geringeren Fallzahl nicht signifikant. In der alternativen Berechnung unter Ausschluss der Doppelmitgliedschaften verschwindet der Zusammenhang zum VIKZ, während die AABF-Mitgliedschaft europäische Netzwerke signifikant begünstigt.

76 Siehe als wichtigen Beitrag hierzu Schrover, Vermeulen 2005.

Leviathan, 40. Jg., 1/2012 
sind, dass Gemeinden religiöse wie auch integrationsrelevante Angebote unterbreiten, Kooperationen mit der Aufnahmegesellschaft eingehen und Strukturen herausbilden, die es ihnen ermöglichen, auf durch intergenerationalen und sozialen Wandel veränderte Bedarfe ihrer Klientel zu reagieren.

Bei denjenigen Aktivitäten, die die Offenheit zur deutschen Gesellschaft und die Sozialintegration der Klientel unterstützen können, wurden keine Merkmale erkennbar, die mit der Umsetzung von Ressourcen in solche Aktivitäten interferieren. Von den großen Glaubensrichtungen oder Verbänden wurden keine als in diesem Sinne »integrationsresistent " kenntlich. ${ }^{77}$ Differenzierter ist die Situation hinsichtlich der Erneuerungsfähigkeit der Gemeinden. Hier erweist sich die Ressourcenausstattung bei den verbandsunabhängigen Gemeinden als besonders bedeutend für die Erreichung Jugendlicher und der Nachfolgegeneration.

Faktisch ist die Bedeutung der großen Verbände für die Bereitstellung religiöser Infrastruktur nicht nur ungebrochen, ihren Gemeinden gelingt es auch, ihre Angebote an sich wandelnde Bedürfnisse anzupassen. Wie ebenso in anderen Befunden feststellbar, ${ }^{78}$ sind unter Gemeindeneugründungen der letzten Jahre zwar verbandsunabhängige Gemeinden überproportional vertreten, als Reaktion auf ein »Versagen « der etablierten Verbände, mit dem Wandel der Klientel Schritt zu halten, lässt sich dies jedoch nicht deuten. Unser Ergebnis bestätigt bereits vorliegende Befunde über dynamische Erneuerungsprozesse in großen Verbänden. ${ }^{79}$

Ein Gegensatz zwischen einem autochthonen, europabezogenen Islam und einer herkunftslandbezogenen Variante wird aus unseren Daten nicht erkennbar. Europäische Diaspora- und Herkunftslandkontakte sind eher selten und stehen dann nicht in einem Konkurrenzverhältnis, sondern treten eher in Kombination auf. Dies ist auch wünschenswert in Bezug auf politische Hoffnungen, dass Reformentwicklungen in Europa Resonanz in der islamischen Welt finden mögen. Dass sich der Wandel des organisierten Islams nicht im theoretisch zu erwartenden Umfang in der Herausbildung von Landesgrenzen überschreitenden Diaspora-Netzwerken niederschlägt, hat wiederum mit der Ressourcenabhängigkeit solcher Kontakte zu tun. Dies unterstützt die Überlegung, dass für ein vertieftes Verständnis der Entwicklung des organisierten Islams in der Diaspora neben dem religiösen und sozialen Wandel der Muslime vermehrt organisationsspezifische Aspekte in den Blickpunkt rücken müssen.

77 Mit Blick auf den VIKZ gilt diese Aussage möglicherweise nur mit Einschränkung; sollte aber überhaupt ein negativer Effekt der VIKZ-Mitgliedschaft auf die Umsetzung von Ressourcen in integrationsrelevante Aktivitäten und Strukturen bestehen, so wäre dieser gering. Die Möglichkeit eines solchen Effekts beruht auf einer methodischen Unsicherheit und ist nicht gewiss.

78 Vgl. Spielhaus 2006.

79 Wie bei Rosenow 2010, S. 196-197, und Schiffauer 2008. 


\section{Literatur}

Alba, Richard; Nee, Victor 2003. Remaking the American mainstream: assimilation and contemporary integration. Cambridge: Harvard University Press.

Allievi, Stefano 2003. "Islam in the Public Space«, in Muslim networks and transnational communities in and across Europe, hrsg. v. Allievi, Stefano; Nielsen, Jorgen, S. 1-27. Leiden: Brill.

Amelina, Anna; Faist, Thomas 2008. "Turkish migrant associations in Germany: between integration pressure and transnational linkages ", in Revue Européenne des Migrations Internationales 2, S. 67-90.

Azzaoui, Mounir 2011. "Muslimische Gemeinschaften in Deutschland zwischen Religionspolitik und Religionsverfassungsrecht - Schieflagen und Perspektiven ", in Politik und Islam, hrsg. v. Schubert, Klaus; Meyer, Hendrik, S. 247-276. Wiesbaden: VS Verlag für Sozialwissenschaften.

Becker, Kip 2004. Islam and business: cross-cultural and cross-national perspectives. Binghamton: International Business Press.

Bertelsmann-Stiftung 2008. Religionsmonitor 2008. Muslimische Religiosität in Deutschland. Überblick über religiöse Einstellungen und Praktiken. Gütersloh: Bertelsmann.

Bundesministerium des Innern. Hrsg. 2011. Deutsche Islam Konferenz. Muslime in Deutschland - deutsche Muslime. Informationsflyer vom 15.08.2001, Artikelnummer BMI07327. Berlin.

Blohm, Michael; Diehl, Claudia 2001. »Wenn Migranten Migranten befragen: Zum Teilnahmeverhalten von Einwanderern bei Bevölkerungsbefragungen ", in Zeitschrift für Soziologie 3, S. 223-242.

Brettfeld, Karin; Wetzels, Peter 2007. Muslime in Deutschland - Integration, Integrationsbarrieren, Religion sowie Einstellungen zu Demokratie, Rechtsstaat und politisch-religiös motivierter Gewalt. Ergebnisse von Befragungen im Rahmen einer multizentrischen Studie in städtischen Lebensräumen. Texte zur Inneren Sicherheit des Bundesministeriums des Innern. Hamburg/Berlin: BMI.

Chbib, Raida 2011. »Einheitliche Repräsentation und muslimische Binnenvielfalt. Eine datengestützte Analyse der Institutionalisierung des Islams in Deutschland «, in Politik und Islam, hrsg. v. Schubert, Klaus; Meyer, Hendrik, S. 87-112. Wiesbaden: VS Verlag für Sozialwissenschaften.

Child, John; Loveridge, Ray; Warner, Malcolm 1973. »Towards an organizational study of trade unions", in Sociology 7, S. 71-91.

Crul, Maurice; Schneider, Jens 2010. »Comparative integration context theory: participation and belonging in new diverse European cities ", in Ethnic and Racial Studies 33, S. 1249-1268.

Diehl, Claudia; Blohm, Michael 2008. »Die Entscheidung zur Einbürgerung. Optionen, Anreize und identifikative Aspekte ", in Migration und Integration. Sonderheft der Kölner Zeitschrift für Soziologie und Sozialpsychologie 48, hrsg. v. Kalter, Frank, S. 437-464. Wiesbaden: VS Verlag für Sozialwissenschaften.

Esser, Hartmut 2008. »Assimilation, ethnische Schichtung oder selektive Akkulturation? Neuere Theorien der Eingliederung von Migranten und das Modell der intergenerationalen Integration ", in Migration und Integration. Sonderheft der Kölner Zeitschrift für Soziologie und Sozialpsychologie 48, hrsg. v. Kalter, Frank, S. 81-107. Wiesbaden: VS Verlag für Sozialwissenschaften.

Esser, Hartmut 2009. »Pluralisierung oder Assimilation? Effekte der multiplen Inklusion auf die Integration von Migranten ", in Zeitschrift für Soziologie 38, S. 358-378.

Fromm, Sabine 2010. Datenanalyse mit SPSS für Fortgeschrittene 2: Multivariate Verfahren für Querschnittsdaten. Wiesbaden: VS Verlag für Sozialwissenschaften.

Halm, Dirk et al. 2012. »TRAMO country study Germany ", in Cross-border migrant organisations in comparative perspective, hrsg. v. Pries, Ludger; Sezgin, Zeynep (im Erscheinen).

Haug, Sonja; Müssig, Stephanie; Stichs, Anja 2009. Muslimisches Leben in Deutschland. BAMF Forschungsbericht 6 . Nürnberg.

Hero, Markus; Krech, Volkhard; Zander, Helmut. Hrsg. 2008. Religiöse Vielfalt in NRW. Empirische Befunde und Perspektiven der Globalisierung vor Ort. Paderborn: Schöningh.

Homeyer, Josef 2007. Leitideen des » bosnischen Islam « als Perspektiven für einen »europäischen Islam «? Beitrag zur Tagung »Bosnischer Islam für Europa « der Akademie der Diözese Rot-

Leviathan, 40. Jg., 1/2012 
tenburg-Stuttgart, 16.-17.11.2007, www.akademie-rs.de/fileadmin/user_upload/download _archive/interreligioeser-dialog/071116_homeyer_leitidee.pdf (Zugriff vom 08.08.2011).

Hunger, Uwe 2005. "Ausländische Vereine in Deutschland. Eine Gesamterfassung auf der Basis des Bundesausländerzentralregisters", in SelbstHilfe. Wie Migranten Netzwerke knüpfen und Soziales Kapital schaffen, hrsg. v. Thränhardt, Dietrich; Weiss, Karin, S. 221-244. Freiburg: Lambertus.

Jonker, Gerdien 2002. Eine Wellenlänge zu Gott. Der» Verband der Islamischen Kulturzentren in Europa«. Bielefeld: transcript.

Karam, Azza. M. Hrsg. 2004. Transnational political Islam: religion, ideology and power. London: Pluto Press.

Klause, Ralph-Peter 2006. Der Islam in der Regio TriRhena. Muslimisches Leben am Oberrhein. Münster: Waxmann.

Koopmans, Ruud 2005. "Migrant claims-making between transnationalism and national citizenship ", in Contested Citizenship, Koopmans, Ruud et al., S. 146-179. Minneapolis: University of Minnesota Press.

Krech, Volkhard 2008. »Religion und Zuwanderung. Die politische Dimension religiöser Vielfalt ", in Religiöse Vielfalt in NR W. Empirische Befunde und Perspektiven der Globalisierung vor Ort, hrsg. v. Hero, Markus; Krech, Volkhard; Zander, Helmut, S. 190-203. Paderborn: Schöningh.

Leggewie, Claus 2009. "Warum es Moscheebaukonflikte gibt und wie man sie bearbeiten kann «, in Moscheen in Deutschland. Religiöse Heimat und gesellschaftliche Herausforderung, hrsg. v. Beinhauer-Köhler, Bärbel; Leggewie, Claus, S. 117-201. München: C.H. Beck.

Lemmen, Thomas 2001. Muslime in Deutschland. Eine Herausforderung für Kirche und Gesellschaft. Baden-Baden: Nomos.

Nielsen, Jorgen 2003. "Transnational Islam and the integration of Islam «, in Muslim networks and transnational communities in and across Europe, hrsg. v. Nielsen, Jorgen; Allievi, Stefano, S. 28-51. Leiden: Brill.

Pettigrew, Thomas; Tropp, Linda 1998. »Does intergroup contact reduce prejudice? Recent metaanalytical findings", in Reducing prejudice and discrimination, hrsg. v. Oskamp, Stuart, S. 93-114. Mahwah: Erlbaum.

Portes, Alejandro 1998. "Social capital: its origins and applications in modern sociology«, in Annual Review of Sociology 24, S. 1-24.

Portes, Alejandro; Guarnizo, Luis E.; Haller, William 2003: »Assimilation and transnationalism. Determinants of transnational political action among contemporary migrants ", in American Journal of Sociology 108, S. 1211-1248.

Portes, Alejandro; Escobar, Cristina; Radford, Alexandria W. 2007. »Immigrant transnational organizations and development: a comparative study ", in International Migration Review 41, S. 242-281.

Pugh, Derek; Hickson, David; Hinings, Christopher 1969. »An empirical taxonomy of structures of work organizations ", in Administrative Science Quarterly 1, S. 115-126.

Rosenow, Kerstin 2010. »Von der Konsolidierung zur Erneuerung. Eine organisationssoziologische Analyse der Türkisch-Islamischen Union der Anstalt für Religion e.V. DITIB «, in Jenseits von »Identität oder Integration «: Grenzen überspannende Migrantenorganisationen, hrsg. v. Pries, Ludger; Sezgin, Zeynep, S. 169-200. Wiesbaden: VS Verlag für Sozialwissenschaften.

Sauer, Martina; Halm, Dirk 2009. Erfolge und Defizite der Integration türkeistämmiger Einwanderer. Entwicklung der Lebenssituation 1999-2008. Wiesbaden: VS Verlag für Sozialwissenschaften.

Schiffauer, Werner 2008. Parallelgesellschaften. Wie viel Wertekonsens braucht unsere Gesellschaft? Für eine kluge Politik der Differenz. Bielefeld: transcript.

Schiffauer, Werner 2010. Nach dem Islamismus. Eine Ethnographie der Islamischen Gemeinschaft Milli Görüs. Berlin: Suhrkamp.

Schrover, Malou; Vermeulen, Floris 2005. »Immigrant Organisations «, in Journal of Ethnic and Migration Studies 5, S. 823-832.

Sökefeld, Martin 2008 a. Struggling for Recognition: The Alevi movement in Germany and in transnational space. Oxford: Berghahn. 
Sökefeld, Martin 2008 b. Aleviten in Deutschland. Identitätsprozesse einer Religionsgemeinschaft in der Diaspora. Bielefeld: transcript.

Sökefeld, Martin 2008 c. » Difficult identifications: the debate on Alevism and Islam in Germany «, in Islam and Muslims in Germany, hrsg. v. Al-Harmaneh, Ala; Thielmann, Jörn, S. 267-297. Leiden: Brill.

Spielhaus, Riem 2006. "Organisationsstrukturen islamischer Gemeinden ", in Islamisches Gemeindeleben in Berlin, hrsg. v. Spielhaus, Riem; Färber, Alexa, S. 12-17. Berlin: Der Beauftragte des Senats von Berlin für Integration und Migration.

Thränhardt, Dietrich et al. 1999. Selbstorganisationen von Migrantinnen und Migranten in NRW. Wissenschaftliche Bestandsaufnahme. Schriftenreihe des MASSKS NRW. Düsseldorf.

Thränhardt, Dietrich; Hunger, Uwe. Hrsg. 2000: Einwanderer-Netzwerke und ihre Integrationsqualität in Deutschland und Israel. Münster: LIT Verlag.

Thränhardt, Dietrich; Weiss, Karin. Hrsg. 2005. SelbstHilfe. Wie Migranten Netzwerke knüpfen und soziales Kapital schaffen. Freiburg: Lambertus.

Tietze, Nikola 2001. Islamische Identitäten. Formen muslimischer Religiosität bei jungen Männern in Deutschland und Frankreich. Hamburg: Hamburger Edition.

Wunn, Ina 2007. Muslimische Gruppierungen in Deutschland. Ein Handbuch. Stuttgart: Kohlhammer. 
Zusammenfassung: Der Beitrag thematisiert die Angebote und Organisationsstrukturen muslimischer einschließlich alevitischer Gemeinden in Deutschland. Auf der Grundlage der Befragung von 1.141 religiösen Organisationen wurden Zusammenhänge intergenerationalen Wandels, des Wandels der muslimischen Organisationslandschaft und der "Integrationsorientierung « der Gemeinden untersucht. Als Ergebnis zeigt sich der organisierte Islam in Deutschland als grundsätzlich wandlungsfähig, wobei die Ressourcenausstattung der Gemeinden sich als zentraler Einflussfaktor für die Angebote der Organisationen erweist: Sie bestimmt den Umfang religiöser und sozialintegrativer Aktivitäten sowie grenzüberschreitender Kooperationen in höherem Maße als die Zugehörigkeit zu bestimmten Verbänden oder Glaubensrichtungen.

Stichworte: Islam in Deutschland - Migrantenorganisationen - Moscheen - Sozialer Wandel - Transnationalismus

\title{
Mosques in Germany: organizational structure and services
}

Summary: In this paper we present a survey of mosques in Germany, including Alevi Cem Houses, focusing in particular on their organizational structures and services. The survey is based on interviews with 1.141 religious organisations; we examined the relationships between intergenerational change, the evolution of organisational landscapes and changes in the services offered by Muslim organizations to meet the demands of their clientele and address matters of social integration. We find that organised Islam in Germany is essentially versatile. Resources are the decisive factor determining what kind of and how many services an organisation may offer, whether it is religious or non-religious. Resources also determine the extent of cross-border and host country activities to a much higher degree than individual religious persuasion or individual membership in specific Muslim associations.

Keywords: Islam in Germany - migrant organisations - mosques - social change - transnationalism

\author{
Autoren \\ PD Dr. Dirk Halm \\ Stiftung ZfTI an der Universität Duisburg-Essen \\ halm@zfti.de \\ Dr. Martina Sauer \\ Stiftung ZfTI an der Universität Duisburg-Essen \\ sauer@zfti.de
}

